hep-th/0103256

\title{
On Noncommutative Multi-solitons
}

\author{
Rajesh Gopakumar, Matthew Headrick and Marcus Spradlin \\ Jefferson Physical Laboratory \\ Harvard University \\ Cambridge MA 02138 \\ E-mail: headrick@physics.harvard.edu
}

\begin{abstract}
We find the moduli space of multi-solitons in noncommutative scalar field theories at large $\theta$, in arbitrary dimension. The existence of a non-trivial moduli space at leading order in $1 / \theta$ is a consequence of a Bogomolnyi bound obeyed by the kinetic energy of the $\theta=\infty$ solitons. In two spatial dimensions, the parameter space for $k$ solitons is a Kähler de-singularization of the symmetric product $\left(\mathbb{R}^{2}\right)^{k} / S_{k}$. We exploit the existence of this moduli space to construct solitons on quotient spaces of the plane: $\mathbb{R}^{2} / \mathbb{Z}_{k}$, cylinder, and $T^{2}$. However, we show that tori of area less than or equal to $2 \pi \theta$ do not admit stable solitons. In four dimensions the moduli space provides an explicit Kähler resolution of $\left(\mathbb{R}^{4}\right)^{k} / S_{k}$. In general spatial dimension $2 d$, we show it is isomorphic to the Hilbert scheme of $k$ points in $\mathbb{C}^{d}$, which for $d>2$ (and $k>3$ ) is not smooth and can have multiple branches.
\end{abstract}

March 2001 


\section{Contents}

1. Introduction . . . . . . . . . . . . . . . . . . . . . . . . . . . . . . . . . 1

2. The moduli space at infinite $\theta$. . . . . . . . . . . . . . . . . . . . . . . . . . . . . . . . 4

2.1. Solitons and projection operators . . . . . . . . . . . . . . . . . . . . . . . 4

2.2. Geometry of the Grassmannian . . . . . . . . . . . . . . . . . . . . . . . 6

3. The moduli space at finite $\theta$. . . . . . . . . . . . . . . . . . . . . . . . . . . . . . . . 0

3.1. The Bogomolnyi bound . . . . . . . . . . . . . . . . . . . . . . . . . . . 7

3.2. Topology . . . . . . . . . . . . . . . . . . . . . . . . . . . . . . . . . 8

3.3. Geometry . . . . . . . . . . . . . . . . . . . . . . . . . . 10

4. The effective potential on the moduli space . . . . . . . . . . . . . . . . . . . . . 14

5. Solitons on quotient spaces . . . . . . . . . . . . . . . . . . . . . . . . . . . 16

5.1. $\mathbb{R}^{2} / \mathbb{Z}_{k}$. . . . . . . . . . . . . . . . . . . . . . . . . . . . . . . . . . 16

5.2. Cylinder . . . . . . . . . . . . . . . . . . . . . . . . . . . 16

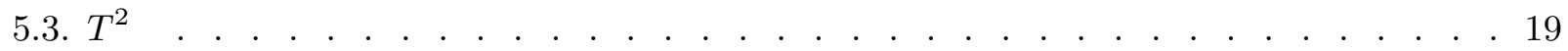

6. Solitons in higher dimensions . . . . . . . . . . . . . . . . . . . . . . . . . . 21

6.1. $4+1$ dimensions . . . . . . . . . . . . . . . . . . . . . . . . . . . . . . 22

6.2. Hilbert schemes of points . . . . . . . . . . . . . . . . . . . . . . . . . . 23

6.3. An exotic example . . . . . . . . . . . . . . . . . . . . . . . . . . . . . . . 24

Appendix A. Completeness and smoothness of the finite $\theta$ moduli space . . . . . . . . 25

A.1. Some elementary machinery . . . . . . . . . . . . . . . . . . . . . . . . . 26

A.2. Completeness . . . . . . . . . . . . . . . . . . . . . . . . . . . . . . . 27

A.3. Smoothness . . . . . . . . . . . . . . . . . . . . . . . . . . . . . . . . 28

Appendix B. Construction of the soliton on the integral torus . . . . . . . . . . . . . 29

\section{Introduction}

Among the many reasons to study noncommutative field theories is that they might be useful for studying stringy behavior in a controlled manner. In the context of string theory, the noncommutativity is a remnant of the noncommutative geometry of open string field theory [1-4]. An interesting class of excitations in the field theory are noncommutative solitons [5]. The existence and form of these classical solutions are fairly independent of the details of the theory, making them useful probes of stringy behavior. Coincident solitons exhibit a non-abelian enhancement of the zero mode spectrum, and in fact these solitons are the D-branes of string theory manifested in a field theory limit while still capturing many of their stringy features. Note that, in contrast, D-branes do not appear as finite energy excitations in conventional (commutative) field theory limits of string theory.

The fact that one can see D-branes in a simpler field theoretic context has had important consequences, primarily in the study of tachyon condensation in open string field 
theory [6,7]. Many of Sen's conjectures [8,9] on this subject have been beautifully confirmed using properties of noncommutative solitons. Similarly, unstable solitons in noncommutative gauge theory [10,11] can be interpreted as codimension two (or higher) D-branes localized on other D-branes [12,13]. The gauge theory successfully reproduces the dynamics of tachyon condensation in this system. Other studies of scalar solitons include 1423 .

In this paper we will begin to investigate some aspects of how D-branes see spacetime by exploring the moduli space of solitons in noncommutative scalar field theory in $2 d+1$ dimension. The study of multi-solitons itself turns out to have many interesting features. At $\theta=\infty$, because of the presence of the $\mathrm{U}(\infty)$ symmetry in the dominant potential energy term, there is an infinite dimensional moduli space of solutions corresponding to arbitrary hermitian projection operators on a Hilbert space $\mathcal{H}$. Since this symmetry is not preserved by the kinetic term, one might expect the degeneracy to be completely lifted when one includes this leading $1 / \theta$ correction. Surprisingly, we find that there remains a smaller yet non-trivial moduli space at this order, which roughly corresponds to individual gaussian solitons free to roam the plane. The lack of a force between static solitons at this order in $1 / \theta$ is due to a Bogomolnyi bound for the kinetic energy of projection operators.

This moduli space, however, is not protected by any symmetry, and is lifted by a classical effective potential which is generated at the next order in $1 / \theta$, and presumably by quantum corrections as well. The classical effective potential (which is bounded both above and below) leads to an attractive force among the solitons. Thus in the true (exact) moduli space all $k$ solitons are coincident. Nevertheless, the approximate moduli space is a useful description of the dynamics of multiple solitons within a certain range of energies [25].

Why is this moduli space interesting if it is only an approximate one? The answer is twofold. Firstly, this moduli space is very closely related to the symmetric product spaces $\left(\mathbb{R}^{2 d}\right)^{k} / S_{k}$, which arise in the study of supersymmetric vacua of $\mathrm{D} p$ - $\mathrm{D} p^{\prime}$ systems in string theory. In fact, we will see that our moduli space (for a scalar field theory with noncommutativity in $2 d$ spatial directions) is precisely the same as the so-called Hilbert scheme of $k$ points in $\mathbb{R}^{2 d}$. This is, for $d \leq 2$, a smooth resolution of the symmetric product space which is potentially singular when points coincide. For the case of $d=2$ we see that the solitons resolve the singularity in an interesting way: when any two of them are brought together, the final configuration depends on the complex direction in the relative $\mathbb{R}^{4}$ by which they approach each other, so that there is a hidden $\mathbb{P}^{1}$ at the 
putative singular point. This is also exactly what D-branes see as a stringy resolution of the singularity. For $d>2$, interestingly, the Hilbert scheme is not a manifold in any sense and has exotic branches which also show up in brane systems. In our context, these branches reflect the fact that coincident solitons in higher dimension have a large number of moduli associated with the shape of the lump solution.

The second reason why these approximate moduli spaces are interesting is that they enable us to construct infinite arrays of solitons in $\mathbb{R}^{2 d}$ that respect some discrete symmetry. Such an array can be viewed as a single soliton on the quotient space. The lowest energy stable solutions on the quotient space - the analogues of the gaussian soliton on $\mathbb{R}^{2}$ - are constructed this way. For the torus we encounter the somewhat unexpected fact that solitons do not exist when the area is less than or equal to $2 \pi \theta$.

The moduli spaces also inherit an interesting geometric structure from the noncommutativity. For instance, in the simplest case of two spatial dimensions, the $k$ soliton moduli space is - as a complex manifold- simply $\operatorname{Sym}^{k}\left(\mathbb{R}^{2}\right) \equiv\left(\mathbb{R}^{2}\right)^{k} / S_{k}$, the symmetric product of the single soliton moduli space $\mathbb{R}^{2}$. Geometrically, however, the solitons smooth out the conical singularities that occur on $\operatorname{Sym}^{k}\left(\mathbb{R}^{2}\right)$ where two or more points come together, as the explicit Kähler metric shows. (The Kähler metric on the moduli space of $k=2$ solitons has appeared in the work of Lindström et al. [24].) Similarly, the smooth Hilbert scheme resolution of $\operatorname{Sym}^{k}\left(\mathbb{R}^{4}\right)$ also inherits a Kähler metric, distinct from the hyperkähler one which arises in the case of instanton physics.

That noncommutative solitons have such a rich structure in their moduli space is very encouraging and makes them worthy of further exploration. The way apparent singularities are resolved is very stringy, and it is interesting that the noncommutative algebra of projection operators sees the resolved geometry in a simple way, which the commutative algebra of functions cannot. This is perhaps a clue that noncommutative algebras might play a fundamental role in understanding geometry in string theory.

Some of the results in this paper were announced at Strings 2001 [39]. The paper [28] by E. Martinec and G. Moore, which has appeared in the meanwhile, has overlap with the discussion in section 5 . K. Lee and collaborators have studied the resolution of moduli spaces of instantons and vortices on noncommutative spaces in 29.31. 


\section{The moduli space at infinite $\theta$}

In this section we briefly review the construction of solitons in $2+1$ dimensional noncommutative scalar field theory at large $\theta$. The moduli space at $\theta=\infty$ (defined precisely below) is isomorphic to the space of projection operatorst on an infinite-dimensional Hilbert space. We review the relevant mathematical details about the geometry of this space (known as the Grassmannian).

\subsection{Solitons and projection operators}

Until the last section of this paper, we work in a $2+1$ dimensional scalar field theory with spacelike noncommutativity:

$$
S=\int d t d^{2} w\left(\frac{1}{2} \dot{\phi}^{2}-\partial_{w} \phi \partial_{\bar{w}} \phi-m^{2} V(\phi)_{\star}\right),
$$

where $w=\left(x^{1}+i x^{2}\right) / \sqrt{2}$. The subscript on the potential indicates that it is evaluated with the Moyal star product, with noncommutativity parameter $\theta^{w \bar{w}}=-i \theta$ (for details, see [5]). It will be convenient to let the factor $m^{2}$ multiply the entire potential, so we assume $V^{\prime \prime}(0)=1$. The existence of stable solitons in this theory depends on the potential having a second, local minimum, which we put at $\phi=\lambda$, with $V(\lambda)>V(0)=0$ (figure 1). The energy functional for static configurations, $E[\phi]=\int d^{2} w\left(\partial_{w} \phi \partial_{\bar{w}} \phi+m^{2} V(\phi)_{\star}\right)$, may be conveniently rewritten using the Weyl-Moyal correspondence, $w \rightarrow \sqrt{\theta} a, \partial_{w} \rightarrow-\frac{1}{\sqrt{\theta}}\left[a^{\dagger}, \cdot\right]$, etc., which maps the star product into operator multiplication in the Hilbert space $\mathcal{H}$ of a 1-dimensional particle:

$$
E\left[\hat{\phi}, \theta m^{2}\right]=2 \pi \operatorname{Tr}_{\mathcal{H}}\left([a, \hat{\phi}]\left[\hat{\phi}, a^{\dagger}\right]+\theta m^{2} V(\hat{\phi})\right) .
$$

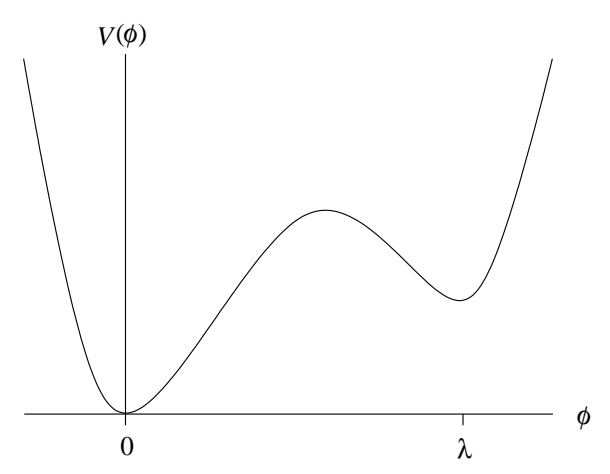

Fig. 1: The potential for $\phi$ is assumed to have a global minimum at $\phi=0$ and a local minimum at $\phi=\lambda$.

1 Throughout this paper, by "projection operator" we mean "hermitian projection operator." 
Fixing the function $V(\phi)$, it is the dimensionless parameter $\theta m^{2}$ that controls the relative importance of the kinetic and potential terms in (2.2). Exact solutions to the equation of motion are known in the limit $\theta m^{2} \rightarrow \infty$, when the kinetic term may be neglected compared to the potential term [5]. In this section we describe the moduli space of such solitons. In the next section we include the kinetic term as a perturbation, and see how it lifts the moduli space down to a much smaller but still non-trivial one.

To define the theory in the limit $\theta \mathrm{m}^{2} \rightarrow \infty$ we must rescale the energy:

$$
E_{0}[\hat{\phi}] \equiv \lim _{\theta m^{2} \rightarrow \infty} \frac{1}{\theta m^{2}} E\left[\hat{\phi}, \theta m^{2}\right]=2 \pi \operatorname{Tr} V(\hat{\phi}) .
$$

Stable solutions to the resulting equation of motion $V^{\prime}(\hat{\phi})=0$ take the form

$$
\hat{\phi}=\lambda P,
$$

where $P$ is any projection operator. The energy of such a solution is $E_{0}=2 \pi k V(\lambda)$, where $k$ is the rank of $P$, so we will assume for now that this rank is finite. (In section 5 we will also discuss projection operators of infinite rank.) If one interprets the rank one solutions as single solitons, then the rank $k$ solutions may be thought of as corresponding to $k$ non-interacting solitons, each of energy $2 \pi V(\lambda)$. This interpretation will become more meaningful in the next section.

Note that (2.3) has an invariance under arbitrary unitary transformations $U$ of the Hilbert space, under which $\hat{\phi} \rightarrow U \hat{\phi} U^{\dagger}$. Since any two projection operators of rank $k$ can be continuously connected by $\mathrm{U}(\infty)$ transformations, there is an infinite dimensional moduli space of solutions with energy $2 \pi k V(\lambda)$. In fact, the rank $k$ projection operators on $\mathcal{H}$ (or equivalently, the $k$-dimensional hyperplanes in $\mathcal{H}$ ) form a manifold known as the Grassmannian $\operatorname{Gr}(k, \mathcal{H})$, which can also be described as the coset space

$$
\frac{\mathrm{U}(\infty)}{\mathrm{U}(k) \times \mathrm{U}(\infty-k)},
$$

where $\mathrm{U}(\infty)$ acts on the entire space, while $\mathrm{U}(\infty-k)$ acts only on the orthogonal complement of a $k$-dimensional hyperplane. The $\mathrm{U}(\infty)$ symmetry protects the moduli space against corrections.

In the next subsection, we describe the geometry of the Grassmannian, partly because of its interest as the moduli space of solitons in the limit $\theta m^{2} \rightarrow \infty$, but more importantly because this will give us the tools to study the geometry of the moduli space of solitons at finite $\theta \mathrm{m}^{2}$, which is a submanifold of the Grassmannian. 


\subsection{Geometry of the Grassmannian}

The Grassmannian has a natural complex structure, which it inherits from $\mathcal{H}$. Points (vectors) in $\mathcal{H}$ may be parametrized by a (infinite) set of holomorphic coordinates $z_{a}$; these could be the coefficients of the vector in some particular basis. If we now have a set of $k$ linearly independent vectors $\left|\psi_{i}\right\rangle \in \mathcal{H}$ which depend holomorphically on the $z_{a}$, then the hyperplane they span is also considered to depend holomorphically on the $z_{a}$.

The Grassmannian also has a natural Kähler structure, which can be computed explicitly as follows 2 Defining the matrix

$$
h_{i j} \equiv\left\langle\psi_{i} \mid \psi_{j}\right\rangle
$$

and its inverse $h^{i j}$, the hermitian operator that projects onto the hyperplane spanned by the $\left|\psi_{i}\right\rangle$ is

$$
P=\left|\psi_{i}\right\rangle h^{i j}\left\langle\psi_{j}\right|
$$

The matrix $h_{i j}$ is the metric on the image of $P$. It is straightforward to show that the metric on the Grassmannian is Kähler,

$$
g_{a \bar{b}} \equiv \operatorname{Tr}\left(\partial_{a} P \partial_{\bar{b}} P\right)=\partial_{a} \partial_{\bar{b}} K
$$

and the Kähler potential is given simply by

$$
K=\ln \operatorname{det}\left(h_{i j}\right) .
$$

There is an ambiguity in choosing the $\left|\psi_{i}\right\rangle$, with any two choices being related by a GL $(k)$ matrix that depends holomorphically on the coordinates. The respective Kähler potentials will differ by a holomorphic plus an anti-holomorphic function, leaving the metric unchanged.

Although the derivation of the moduli space metric has been presented as a mathematical triviality, we emphasize that the physical moduli space metric, which arises as the kinetic term for time-dependent moduli $z_{a}(t)$, differs from (2.8) only by an overall factor. After rescaling the energy as in (2.3), we find the physical metric

$$
\frac{1}{\theta m^{2}} \int d^{2} w \partial_{a} \phi \partial_{\bar{b}} \phi=\frac{2 \pi \lambda^{2}}{m^{2}} \operatorname{Tr}\left(\partial_{a} P \partial_{\bar{b}} P\right)=\frac{2 \pi \lambda^{2}}{m^{2}} \partial_{a} \partial_{\bar{b}} K,
$$

with $K$ given by the formula (2.9).

2 For the mathematical cognoscenti: the natural Kähler form may be obtained as the curvature of a certain line bundle. Let $E$ be the tautological bundle whose fiber over a point in $\operatorname{Gr}(k, \mathcal{H})$ is simply the $k$-dimensional hyperplane that it is. The inner product $\langle\cdot \mid \cdot\rangle$ on $E$ induces a natural metric on the determinant bundle $\operatorname{det}(E)$ : the norm of a section $\psi=\left|\psi_{1}\right\rangle \wedge \cdots \wedge\left|\psi_{k}\right\rangle$ of $\operatorname{det}(E)$ is simply $\|\psi\|^{2}=\operatorname{det}\left(\left\langle\psi_{i} \mid \psi_{j}\right\rangle\right)$. The curvature form $i \partial_{a} \partial_{\bar{b}} \ln \operatorname{det}\left(\left\langle\psi_{i} \mid \psi_{j}\right\rangle\right)$ of this bundle is the natural Kähler form on $\operatorname{Gr}(k, \mathcal{H})$. 


\section{The moduli space at finite $\theta$}

The $\mathrm{U}(\infty)$ symmetry that protects the moduli space in the limit $\theta m^{2} \rightarrow \infty$ is broken in the scalar theory at finite $\theta m^{2}$ by the kinetic term. One might expect that the infinite dimensional moduli space $\operatorname{Gr}(k, \mathcal{H})$ discussed in the previous section is completely lifted at finite $\theta$. Remarkably, we find that despite the lack of symmetry, the leading $1 /\left(\theta m^{2}\right)$ correction to the energy satisfies a Bogomolnyi-like bound which preserves a finite dimensional subspace of the space of projection operators of rank $k$. We show that the remaining moduli space $\mathcal{M}_{k}$ corresponds to $k$ individual solitons which are free to roam the plane, and that $\mathcal{M}_{k}$ has a Kähler metric which is smooth even when the solitons come together.

\subsection{The Bogomolnyi bound}

In a perturbation expansion in $1 /\left(\theta m^{2}\right)$,

$$
\begin{aligned}
& \hat{\phi}=\hat{\phi}_{0}+\frac{1}{\theta m^{2}} \hat{\phi}_{1}+\cdots, \\
& E=\theta m^{2} E_{0}+E_{1}+\frac{1}{\theta m^{2}} E_{2}+\cdots,
\end{aligned}
$$

the first correction to the energy is just the kinetic term:

$$
E_{1}\left[\hat{\phi}_{0}\right]=2 \pi \operatorname{Tr}\left[a, \hat{\phi}_{0}\right]\left[\hat{\phi}_{0}, a^{\dagger}\right] .
$$

Due to the fact that $V^{\prime}\left(\hat{\phi}_{0}\right)=0, E_{1}$ is independent of the correction $\hat{\phi}_{1}$. $E_{1}$ will act like a potential on the space of minima of $E_{0}$ described in the previous section. The minima of this potential will form the moduli space at finite $\theta \mathrm{m}^{2}$, which may then be further corrected at higher orders in $1 /\left(\theta m^{2}\right)$.

A reasonable guess would be that a minimum of the kinetic energy is achieved only by rotationally symmetric solutions. Rotations (about the origin, for simplicity) are generated by the harmonic oscillator Hamiltonian $a^{\dagger} a+1 / 2$, so rotational symmetry translates, for operators, into being diagonal in a basis of harmonic oscillator eigenstates $|n\rangle$. Indeed, it was shown in [5] that any sum of operators of the form $\lambda|n\rangle\langle n|$ extremizes $E_{1}$ (within the infinite $\theta m^{2}$ moduli space), while the operator

$$
\lambda \sum_{n=0}^{k-1}|n\rangle\langle n|
$$


was conjectured to minimize it. 3 In particular, for $k=1$ this would be achieved by the gaussian soliton $\lambda|0\rangle\langle 0|$. Using the (exact) translational symmetry of the theory to center such a solution at any point on the plane, the moduli space (for any given $k$ ) appears to be simply the plane itself, and the moduli space dynamics completely trivial.

However, the story is not so simple. It turns out that there are non-rotationally symmetric minima of $E_{1}$. Indeed, the full moduli space $\mathcal{M}_{k}$ has an interesting structure, large enough to allow non-trivial dynamics. This unexpected fact is a consequence, not of any symmetry possessed by $E_{1}$, but rather of a Bogomolnyi-like bound that it satisfies:

$$
E_{1}\left[\hat{\phi}_{0}\right]=2 \pi \lambda^{2} \operatorname{Tr}[a, P]\left[P, a^{\dagger}\right]=2 \pi \lambda^{2} \operatorname{Tr}\left(P+2 F(P)^{\dagger} F(P)\right) \geq 2 \pi \lambda^{2} k
$$

where

$$
F(P) \equiv(1-P) a P
$$

The bound is saturated when $F(P)=0$, in other words when the image of $P$ is an invariant subspace of the operator $a$. The projection operators satisfying this condition define a subspace $\mathcal{M}_{k}$ of the Grassmannian $\operatorname{Gr}(k, \mathcal{H})$. In the next subsection we will see that it is a finite dimensional subspace, and that the field configurations corresponding to these projection operators have a natural interpretation in terms of separated solitons. In the following subsection we will then investigate the geometry of $\mathcal{M}_{k}$, showing in particular that it is smooth.

\subsection{Topology}

Starting with the simplest case of $k=1$, it is clear that any 1-dimensional invariant subspace of $a$ must be spanned by an eigenstate of that operator, i.e. by a coherent state. We use the non-normalized coherent states $|z\rangle \equiv e^{z a^{\dagger}}|0\rangle$, their virtue for our purposes being that they depend holomorphically upon the eigenvalue $z$, so that they form a complex submanifold of $\mathcal{H}$. This in turn implies that the moduli space $\mathcal{M}_{1}$ is a (1-dimensional) complex submanifold of $\operatorname{Gr}(1, \mathcal{H})$. It is therefore Kähler, and in fact the metric (2.8) is simply $d s^{2}=d z d \bar{z}$. The solution

$$
\hat{\phi}_{z}=\lambda \frac{|z\rangle\langle z|}{\langle z \mid z\rangle}
$$

3 The authors of [5] showed that $\lambda|0\rangle\langle 0|$ indeed minimizes $E_{1}$, and that infinitesimal unitary transformations which mix $|0\rangle$ and $|1\rangle$ are zero modes of $E_{1}$ around that extremum, thus establishing the conjecture for $k=1,2$.

4 A similar equation has arisen in a different context in 40 . 
maps back under the Weyl-Moyal correspondence to the function

$$
\phi_{z}(w, \bar{w})=2 \lambda e^{-2|w / \sqrt{\theta}-z|^{2}}
$$

This is just a translated gaussian soliton localized around $w=\sqrt{\theta} z$. Its moduli space is naturally isomorphic to the physical plane.

At higher $k$, the most obvious way to construct an invariant subspace of $a$ is as a direct sum of such 1-dimensional invariant subspaces, each spanned by a different coherent state $\left|z_{i}\right\rangle$. We can think of this as $k$ solitons, each with independent collective coordinate $z_{i}$. (Indeed, if the $z_{i}$ are far from each other, then the respective coherent states are nearly orthogonal and the corresponding field configuration is approximately the sum of distant Gaussian solitons (3.7).) The moduli space is, at least naively, the $k$-fold symmetric product of the single-soliton moduli space, $\operatorname{Sym}^{k}(\mathbb{C}) \equiv \mathbb{C}^{k} / S_{k}$ (symmetric because permuting the $z_{i}$ leaves the configuration unchanged; the solitons are like identical particles). However, such symmetric products can be singular at the locus of coincidence, and need to be looked at with care.

Let us consider next the case of $k=2$ and see what happens when two solitons are brought together. The description of the corresponding projection operator in terms of the subspace spanned by coherent states $\left\{\left|z_{1}\right\rangle,\left|z_{2}\right\rangle\right\}$ becomes bad since it seems as if we have only one independent vector at the coincident point. Actually, as $z_{2}$ approaches $z_{1}$, it is not the subspace that becomes singular but simply our description of it. Instead we should describe it as spanned, for example, by the vectors $\left|z_{1}\right\rangle$ and $\left(\left|z_{2}\right\rangle-\left|z_{1}\right\rangle\right) /\left(z_{2}-z_{1}\right)$. This basis has the virtue that it is non-singular in the limit $z_{2} \rightarrow z_{1}$; in fact the second vector goes to $\partial_{z_{1}}\left|z_{1}\right\rangle=a^{\dagger}\left|z_{1}\right\rangle$. Now it is clear that we are still in the moduli space in this limit, since the limiting plane, spanned by $\left|z_{1}\right\rangle$ and $a^{\dagger}\left|z_{1}\right\rangle$, is obviously an invariant subspace of $a$. It is straightforward to generalize this proof for any number $n$ of solitons coming together. In appendix A we show that

$$
\lim _{z_{i} \rightarrow z} \operatorname{span}\left\{\left|z_{1}\right\rangle, \ldots,\left|z_{n}\right\rangle\right\}=\operatorname{span}\left\{|z\rangle, a^{\dagger}|z\rangle, \ldots,\left(a^{\dagger}\right)^{n-1}|z\rangle\right\}
$$

Again, the right hand side is obviously an invariant subspace of $a$.

Conversely, one can see that any finite dimensional invariant subspace of $a$ is a direct sum of spaces of the form (3.8), simply by writing the restriction of $a$ to the subspace in Jordan form. That the moduli space $\mathcal{M}_{k}$ does not degenerate in any way at the coincidence locus is a reflection of the mathematical statement that $\operatorname{Sym}^{k}(\mathbb{C})$ is smooth everywhere. 
The coordinates $z_{i}$ are bad coordinates near the coincidence locus but in fact $\operatorname{Sym}^{k}(\mathbb{C})$ is isomorphic to $\mathbb{C}^{k}$.

Moreover, $\mathcal{M}_{k}$ is isomorphic to $\mathbb{C}^{k}$ as a complex manifold since the spanning vectors $\left|z_{i}\right\rangle$ depend holomorphically on the coordinates $z_{i}$. The isomorphism of complex structures extends to the coincidence locus since the change of basis matrix used in appendix A to go to the non-singular description also depends holomorphically on the $z_{i}$. This also implies that $\mathcal{M}_{k}$ is a complex submanifold of $\operatorname{Gr}(k, \mathcal{H})$.

It is straightforward, using (2.7) and the inverse Weyl-Moyal transformation, to find the physical field configuration $\phi(w, \bar{w})$ corresponding to any given point in $\mathcal{M}_{k}$. For example, with the $z_{i}$ all distinct, we have

$$
\phi(w, \bar{w})=2 \lambda \sum_{i, j=1}^{k} h_{i j} h^{j i} e^{-2\left(\bar{w} / \sqrt{\theta}-\bar{z}_{i}\right)\left(w / \sqrt{\theta}-z_{j}\right)} .
$$

Figure 2 illustrates how the process of two solitons coming together appears in the real space, while figure 3 shows an example of a quadruple soliton.

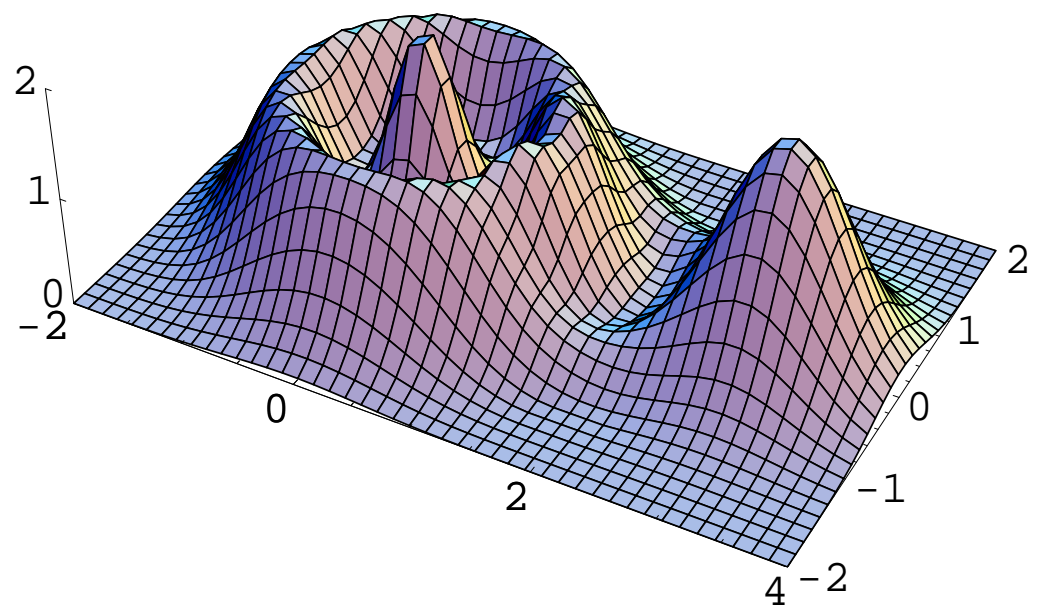

Fig. 3: A quadruple soliton consisting of a triple soliton at the origin and a single soliton at $z=2$. The axes are as in figure 2 .

\subsection{Geometry}

The flat metric $d s^{2}=d z_{i} d \bar{z}_{i}$ on $\operatorname{Sym}^{k}(\mathbb{C})$ has conical singularities on the coincidence locus. The solitons are not pointlike, however, and the smoothness of the merging process 

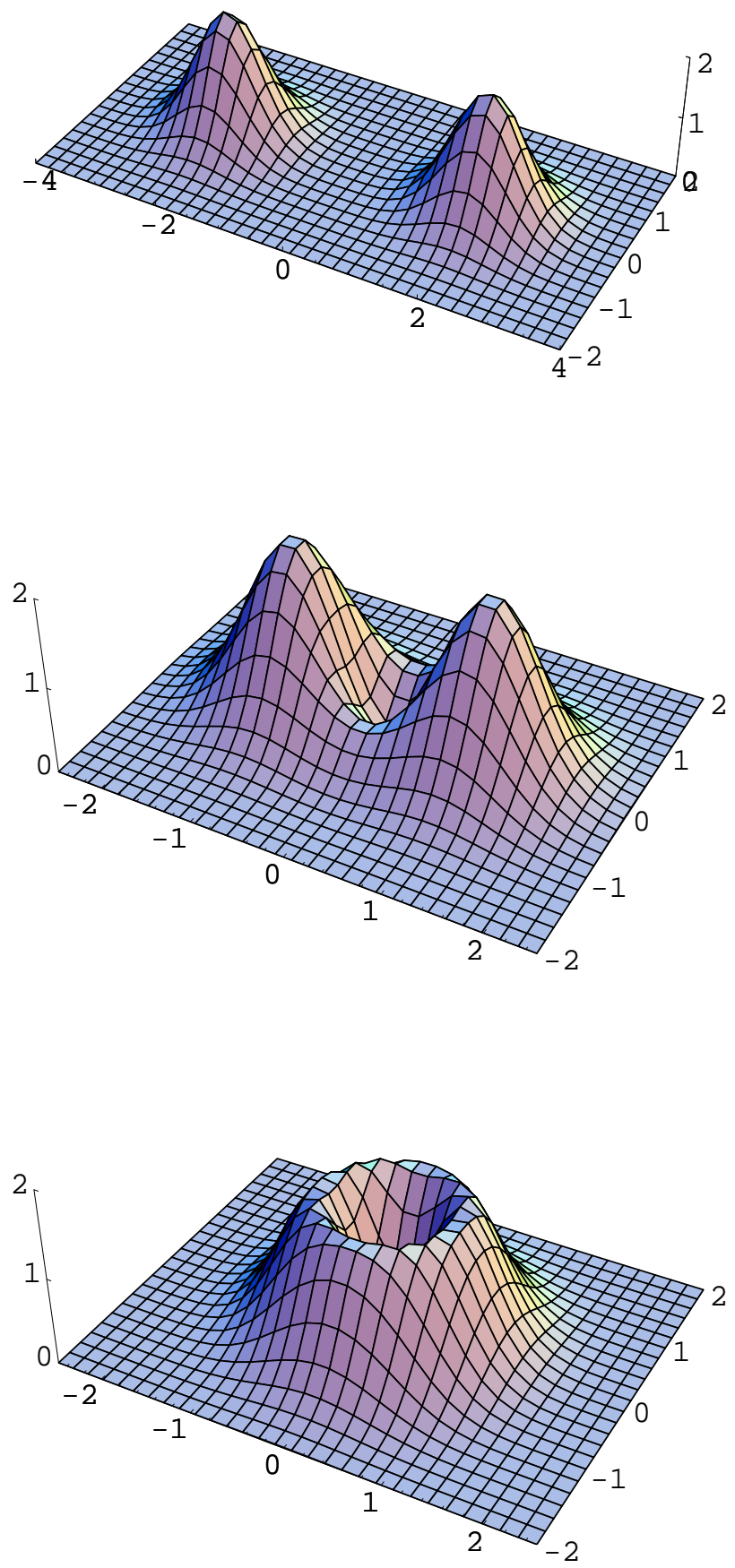

Fig. 2: The double soliton at various separations. Top: At large separation (here $z= \pm 2$ ), the solitons have the same shape as single solitons (3.7). Middle: As they come together they begin to coalesce (here $z= \pm 1$ ). Bottom: When they coincide they form a rotationally symmetric "level 2 " soliton. The vertical axis is $\phi(w, \bar{w}) / \lambda$ and the horizontal plane is $w / \sqrt{\theta}$. 
depicted in figure 2 suggests that they somehow round out these singularities. Indeed, the moduli space $\mathcal{M}_{k}$ has a smooth Kähler metric on it. In this subsection we make some general observations about the Kähler metric and investigate in detail the simplest case of $k=2$ (which has appeared in [24]), but the general proof of smoothness is relegated to appendix A.

The fact that $\mathcal{M}_{k}$ is a complex submanifold of the Kähler manifold $\operatorname{Gr}(k, \mathcal{H})$ implies that $\mathcal{M}_{k}$ itself is Kähler. The Kähler potential is given by (2.9):

$$
K=\ln \operatorname{det}\left(\left\langle z_{i} \mid z_{j}\right\rangle\right)=\ln \operatorname{det}\left(e^{\bar{z}_{i} z_{j}}\right) .
$$

(There is an overall factor of $2 \pi \theta \lambda^{2}$ in the physical moduli space metric, which we will ignore here.)

Two important features of the geometry are immediate consequences of this form for Kähler potential. Firstly, as the translational symmetry of the field theory implies, the center of mass coordinate $c \equiv \frac{1}{k} \sum_{i} z_{i}$ factors out, and the geometry for this modulus is the flat plane:

$$
K=k|c|^{2}+\ln \operatorname{det}\left(e^{\bar{y}_{i} y_{j}}\right)
$$

where $y_{i}=z_{i}-c$ are the relative coordinates. Secondly, when the separations $\left|z_{i}-z_{j}\right|$ are large, the determinant is dominated by the diagonal, so the metric reduces to the flat one on $\operatorname{Sym}^{k}(\mathbb{C})$ :

$$
K \approx \sum_{i=1}^{k}\left|z_{i}\right|^{2} \quad\left(\left|z_{i}-z_{j}\right| \gg 1\right) .
$$

A third property of the Kähler potential is also apparent from equation (3.10): it diverges on the coincidence locus. This is merely a coordinate singularity. The problem is the same as the one we faced in the last subsection: the basis of coherent states $\left|z_{i}\right\rangle$ is degenerate when two or more of the $z_{i}$ coincide. The solution is also the same: make a holomorphic change of basis to a non-degenerate basis. We show in appendix $\mathrm{A}$ that the Jacobian of this change of basis is the Vandermonde determinant $\prod_{i>j}\left(z_{i}-z_{j}\right)$, and when the Kähler potential (3.10) is calculated in this basis one obtains

$$
K^{\prime}=K-\sum_{i>j} \ln \left|z_{i}-z_{j}\right|^{2}
$$

The Kähler potentials $K^{\prime}$ and $K$ yield the same metric away from the coincidence locus, but only the metric obtained from the correct potential $K^{\prime}$ extends smoothly to the locus. 
Let us see in detail how this works in the case of $k=2$. Since the center of mass coordinate plays no role, we will put the two solitons at $z_{1}=z$ and $z_{2}=-z$ respectively. But $z$ is not a good coordinate in the neighborhood of $z=0$, due to the identification $z \sim-z$. The good coordinate is instead $\sigma=z^{2}$, in terms of which the metric is perfectly well behaved near $\sigma=0$ :

$$
K^{\prime}=\ln \left(\frac{2 \sinh 2|z|^{2}}{4|z|^{2}}\right)=\frac{2}{3}|\sigma|^{2}+\mathcal{O}\left(|\sigma|^{4}\right)
$$

SO

$$
d s^{2}=\left(\frac{2}{3}+\mathcal{O}\left(|\sigma|^{2}\right)\right) d \sigma d \bar{\sigma}
$$

The space retains the symmetry of the cone, as well as its geometry far from the vertex, but the vertex itself is rounded out.

The fact that the moduli space is curved implies that there are velocity-dependent forces among the solitons. 0 One can use the metric derived from (3.13) to study the motion of these solitons on the plane, by calculating geodesics on the moduli space [25]. For instance, two-soliton scattering at zero impact parameter has been shown to lead to ninety degree deflection [24]. This can be understood as an immediate consequence of the smoothness and rotational symmetry of the moduli space: A process where two solitons start at $z= \pm z_{0}$ and move toward each other is represented in the moduli space by starting at the point $\sigma=z_{0}^{2}$ and moving toward the origin. The system will pass smoothly through the origin, and by symmetry then pass through the point $\sigma=-z_{0}^{2}$, representing the configuration where the solitons are at $z= \pm i z_{0}$.

For $k>2$ the metric is complicated and therefore difficult to study, but one interesting phenomenon which appears is that the velocity-dependent forces tend to spread coincident solitons apart when they move in the presence of other solitons. For example, if a double soliton moves in the presence of a stationary third soliton, the pair will spread apart along the direction of their motion.

5 Note added in revised version: In the paper [41] (which appeared after the first version of this paper was submitted to the archive), the question was raised as to whether there are boundstate wave-functions due to the curvature of the moduli space. At least for the case $k=2$, we may answer this question in the negative. By using a Weyl transformation to bring the relative moduli space to the plane, it is straightforward to show that the eigenvalue equation for the laplacian admits no normalizable solutions. 


\section{The effective potential on the moduli space}

The moduli space described in the previous section is not protected by any symmetry and is therefore lifted, by classical and presumably quantum effects. A classical effective potential on the moduli space arises from the fact that the solitons $\hat{\phi}_{0}$ we have been discussing so far are not exact solutions, but receive $\mathcal{O}\left(1 /\left(\theta m^{2}\right)\right)$ corrections (the term $\hat{\phi}_{1}$ in (3.1)). We will calculate these corrections explicitly in this section and show that the effective potential leads to an attractive force among the solitons. As we will see, this potential, in addition to being parametrically suppressed, is also well behaved. It is bounded both from below and from above and hence is a small perturbation (for large $\left.\theta m^{2}\right)$ to the moduli space that we studied in the previous section.

In the perturbation series (3.1) for the energy, the first sub-leading correction is

$$
E_{2}\left[\hat{\phi}_{0}, \hat{\phi}_{1}\right]=2 \pi \operatorname{Tr}\left(2\left[a, \hat{\phi}_{0}\right]\left[\hat{\phi}_{1}, a^{\dagger}\right]+\frac{1}{2} \hat{\phi}_{1}^{2} V^{\prime \prime}\left(\hat{\phi}_{0}\right)\right) .
$$

(We have eliminated the term involving $\hat{\phi}_{2}$ due to the vanishing of $V^{\prime}\left(\hat{\phi}_{0}\right)$.) Fixing $\hat{\phi}_{0}$, the equation of motion for $\hat{\phi}_{1}$ obtained from (4.1) is easily solved:[

$$
\hat{\phi}_{1}^{\mathrm{c}}=-2\left[a^{\dagger},\left[a, \hat{\phi}_{0}\right]\right] V^{\prime \prime}\left(\hat{\phi}_{0}\right)^{-1} \text {. }
$$

Plugging this solution back into (4.1), we find, after some algebra, that the correction to the energy is

$$
V_{\text {eff }}(P) \equiv \frac{1}{\theta m^{2}} E_{2}\left[\hat{\phi}_{0}, \hat{\phi}_{1}^{\mathrm{c}}\left(\hat{\phi}_{0}\right)\right]=-\mathcal{E} \operatorname{Tr} G(P)^{2}
$$

where

$$
\mathcal{E} \equiv \frac{4 \pi \lambda^{2}}{\theta m^{2}}\left(1+\frac{1}{V^{\prime \prime}(\lambda)}\right)
$$

and

$$
G(P) \equiv P a(1-P) a^{\dagger} P=P+\left[P a^{\dagger}, a P\right] .
$$

6 We have ignored the issue of operator ordering, which is justified a posteriori by the fact that (as one can readily verify) the solution $\hat{\phi}_{1}^{\mathrm{c}}$ commutes with $\hat{\phi}_{0}$. There is in fact a continuous family of solutions to the correct equation of motion $2\left[a^{\dagger},\left[a, \hat{\phi}_{0}\right]\right]+\left(\hat{\phi}_{1} V^{\prime \prime}\left(\hat{\phi}_{0}\right)\right)_{\mathrm{s}}=0$ (where the subscript $\mathrm{s}$ indicates symmetrization over orderings of $\hat{\phi}_{1}$ and $\left.\hat{\phi}_{0}\right)$, of which $\hat{\phi}_{1}^{\mathrm{c}}$ is the unique one that commutes with $\hat{\phi}_{0}$. The other solutions differ from $\hat{\phi}_{1}^{\mathrm{c}}$ by an operator of the form $P B(1-P)+(1-P) B^{\dagger} P$ for some $B$. One way to understand this is that operators of this form perturb the solution $\hat{\phi}_{0}$ in an "angular" direction (parallel to the infinite $\theta$ moduli space), and the second derivatives of $\operatorname{Tr} V$ vanish in those directions. 
The first way of writing $G(P)$ makes it obvious that it is non-negative definite, while the second way makes it obvious that $\operatorname{Tr} G(P)=k$. Together these two facts imply that $\operatorname{Tr} G(P)^{2}$ is bounded below by $k$ and above by $k^{2}$, i.e. the effective potential is bounded above by $-k \mathcal{E}$ and below by $-k^{2} \mathcal{E}$. The upper bound is achieved when $G(P)=P$, i.e. $\left[P a^{\dagger}, a P\right]=0$; this occurs only in the limit that the solitons are infinitely far from each other, since only in this limit is $a P$ unitarily diagonalizable.

The lower bound, on the other hand, is achieved when $G(P)$ has only a single nonzero eigenvalue, equal to $k$. Let the corresponding eigenvector be $|\psi\rangle$, so that $G(P)=k|\psi\rangle\langle\psi|$. Then for every $\left|\psi^{\prime}\right\rangle$ orthogonal to $|\psi\rangle$,

$$
(1-P) a^{\dagger} P\left|\psi^{\prime}\right\rangle=0,
$$

which in turn implies that the image of $P$ is of the form of the right-hand side of (3.8). (Otherwise, if the image of $P$ were the direct sum of two or more spaces of this form, then every linear combination of the states $\left(a^{\dagger}\right)^{n_{1}-1}\left|z_{1}\right\rangle$ and $\left(a^{\dagger}\right)^{n_{2}-1}\left|z_{2}\right\rangle$ would violate (4.6). Then $G(P)$ would not be of the desired form because it would have two nonzero eigenvalues.) So the effective potential is minimized by configurations in which all $k$ solitons are coincident. The moduli space of such configurations is obviously just the plane itself. In other words, all the moduli $y_{i}$ for relative motion are lifted. The remaining center-ofmass modulus $c$ is exact by the translational symmetry of the theory, and cannot be lifted at any order in $1 /\left(\theta m^{2}\right)$, or by quantum corrections.

The two-soliton effective potential, in terms of the coordinate $\sigma=\left(z_{1}-z_{2}\right)^{2} / 4$, is

$$
V_{\text {eff }}(\sigma)=-2 \mathcal{E}\left(1+\frac{4|\sigma|^{2}}{\sinh ^{2}(2|\sigma|)}\right),
$$

which is plotted in figure 4 . The force is attractive, but falls off exponentially when the solitons are more than a few multiples of $\sqrt{\theta}$ apart. At higher $k$, the functional form of $V_{\text {eff }}$ is more complicated, but it retains these features.

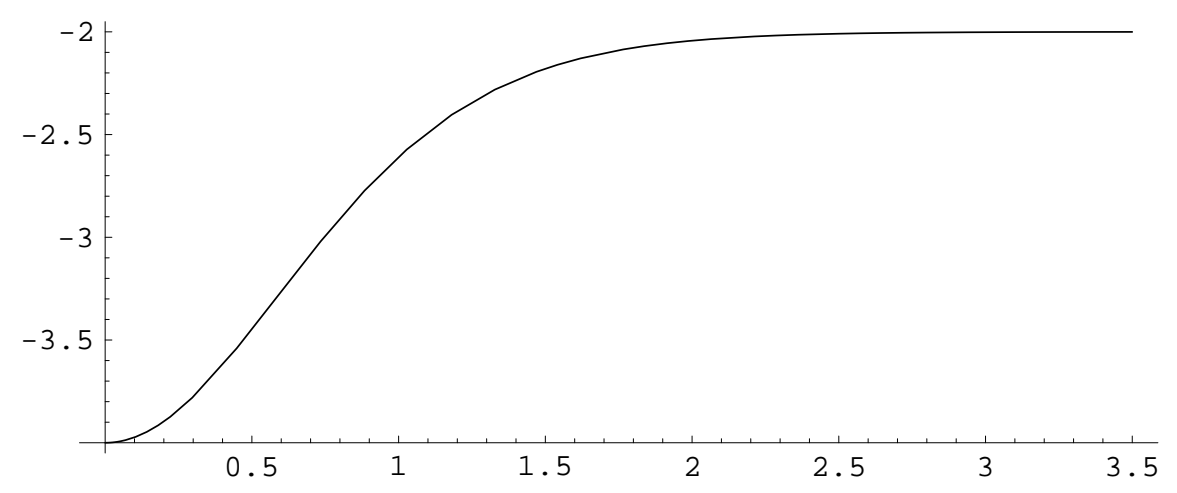

Fig. 4: The two-soliton effective potential (4.7): $V_{\text {eff }} / \mathcal{E}$ versus $|\sigma|=$ $\left|z_{1}-z_{2}\right|^{2} / 4$. 


\section{Solitons on quotient spaces}

We can exploit the results of section 3 for multi-solitons to construct solitons on quotients of $\mathbb{R}^{2}$ by various discrete symmetry groups. The basic principle is simple: construct a multi-soliton on the covering space that respects the quotienting symmetry.

\section{1. $\mathbb{R}^{2} / \mathbb{Z}_{k}$}

As a simple example, consider the orbifold $\mathbb{R}^{2} / \mathbb{Z}_{k}$. We may put $k$ solitons at the vertices of a regular polygon: $z_{i}=\omega^{i} z_{0}$, where $\omega \equiv e^{2 \pi i / k}$. Such configurations form a submanifold of $\mathcal{M}_{k}$, parametrized by the single modulus $z_{0}$; by the rotational symmetry of the theory this submanifold is totally geodesic. This 2-dimensional moduli space is the same orbifold $\mathbb{R}^{2} / \mathbb{Z}_{k}$, since $z_{0}$ is identified with $\omega z_{0}$. Geometrically, however, its Kähler structure is deformed, with the conical singularity at the orbifold fixed point smoothed out, as described in subsection 3.3. This is an example of the stringy behavior of noncommutative solitons: as non-local probes, they see a singular geometry in a non-singular way. The attractive potential of order $\lambda^{2} /\left(\theta m^{2}\right)$ between the soliton and its images, described in section 4, will draw it toward the fixed point.

\subsection{Cylinder}

A soliton on a cylinder of circumference $\sqrt{\theta} l$ can be represented as an infinite array of solitons on the plane, located at $z_{j}=z_{0}+i j l / \sqrt{2}, j \in \mathbb{Z}$, where $z_{0}$ is the modulus. Since the moduli space is quotiented under the identification $z_{0} \sim z_{0}+i l / \sqrt{2}$, it has the same geometry as the underlying cylinder. Furthermore, this moduli space is exact by the translational symmetry of the theory - the attraction between the soliton and its images on one side balance the attraction of those on the other side.

In finding the explicit field configuration $\phi(w, \bar{w})$ of this soliton, equation (3.9) is not of much use, since it requires inverting an infinite dimensional matrix. We will therefore present an alternative construction. For simplicity we will assume in what follows that $z_{0}=$ 0 . It will be convenient to use real coordinates $y^{a} \equiv x^{a} / \sqrt{\theta}\left(\right.$ recall that $\left.w=\left(x^{1}+i x^{2}\right) / \sqrt{2}\right)$, and the corresponding hermitian operators $\hat{y}^{a}$. The discrete translations by which the plane is quotiented are generated by the unitary operator $U \equiv e^{i l \hat{y}^{1}}$. We thus wish to construct the projection operator whose image is spanned by the set $\left\{U^{j}|0\rangle: j \in \mathbb{Z}\right\}$ of (normalized) coherent states. To do this, we will find an orthonormal basis for this hyperplane of the form $\left\{U^{j}|\psi\rangle: j \in \mathbb{Z}\right\}$, where

$$
\left\langle\psi\left|U^{j}\right| \psi\right\rangle=\delta_{j 0}
$$


This will allow us to decompose $P$ as a sum of orthogonal projection operators:

$$
P=\sum_{j} U^{j}|\psi\rangle\langle\psi| U^{-j}
$$

The inverse Weyl-Moyal transformation then yields

$$
\phi\left(y^{1}, y^{2}\right)=\frac{2 \pi \lambda}{l} \sum_{j} e^{-2 \pi i j y^{2} / l} \psi^{*}\left(y^{1}-\frac{\pi j}{l}\right) \psi\left(y^{1}+\frac{\pi j}{l}\right),
$$

where $\psi\left(y^{1}\right) \equiv\left\langle y^{1} \mid \psi\right\rangle$ is the wave function of $|\psi\rangle$ in the basis of $\hat{y}^{1}$ eigenstates.

We will find the vector $|\psi\rangle$ by finding its coefficients in the $U^{j}|0\rangle$ basis, or more precisely, the Fourier transform of those coefficients:

$$
|\psi\rangle=\sum_{j} c_{j} U^{j}|0\rangle=\tilde{c}\left(l \hat{y}^{1}\right)|0\rangle .
$$

Working in terms of the wave functions, with

$$
\psi_{0}\left(y^{1}\right) \equiv\left\langle y^{1} \mid 0\right\rangle=\pi^{-1 / 4} e^{-\left(y^{1}\right)^{2} / 2},
$$

we impose the orthonormality condition (5.1),

$$
\begin{aligned}
\delta_{j^{\prime} 0} & =\int d y^{1} e^{i j^{\prime} l y^{1}}\left|\tilde{c}\left(l y^{1}\right)\right|^{2} \psi_{0}\left(y^{1}\right)^{2} \\
& =\int_{0}^{2 \pi / l} d y^{1} e^{i j^{\prime} l y^{1}}\left|\tilde{c}\left(l y^{1}\right)\right|^{2} \sum_{j} \psi_{0}\left(y^{1}+\frac{2 \pi j}{l}\right)^{2} .
\end{aligned}
$$

We can now solve for $\tilde{c}$ (choosing it to be real) and thus for $\psi$ :

$$
\begin{aligned}
\psi\left(y^{1}\right) & =\sqrt{\frac{l}{2 \pi \sum_{j}\left|\psi_{0}\left(y^{1}+2 \pi j / l\right)\right|^{2}}} \psi_{0}\left(y^{1}\right) \\
& =\sqrt{\frac{l}{2 \pi \vartheta_{00}\left(2 i y^{1} / l, \tau\right)}}
\end{aligned}
$$

where $\tau \equiv 4 \pi i / l^{2}$.

$$
\phi\left(y^{1}, y^{2}\right)=\lambda\left(\frac{\vartheta_{00}\left(2 y^{2} / l, \tau\right)}{\vartheta_{00}\left(2 i y^{1} / l, \tau\right)}+\frac{\vartheta_{10}\left(2 y^{2} / l, \tau\right)}{\vartheta_{10}\left(2 i y^{1} / l, \tau\right)}\right) .
$$

This is plotted in figure 5 for various values of $l$. One can show by a modular transformation that in the decompactification limit $l \rightarrow \infty$, (5.8) goes over to the single soliton on the plane.

7 In fact, this construction is a very general one, applicable to constructing an arbitrary soliton on the cylinder at $\theta=\infty$. In other words we can construct arbitrary projection operators of the form (5.2) which respect the discrete translation symmetry. All one needs to do is replace $|0\rangle$ in (5.4) and (5.5) by an arbitrary ket $|\tilde{\psi}\rangle$. The solution for the coefficients $\tilde{c}\left(l \hat{y}^{1}\right)$ is then as in the first line of (5.7). Examples of such general solitons on the cylinder have also been constructed by V. Balasubramaniam and A. Naqvi (unpublished). 

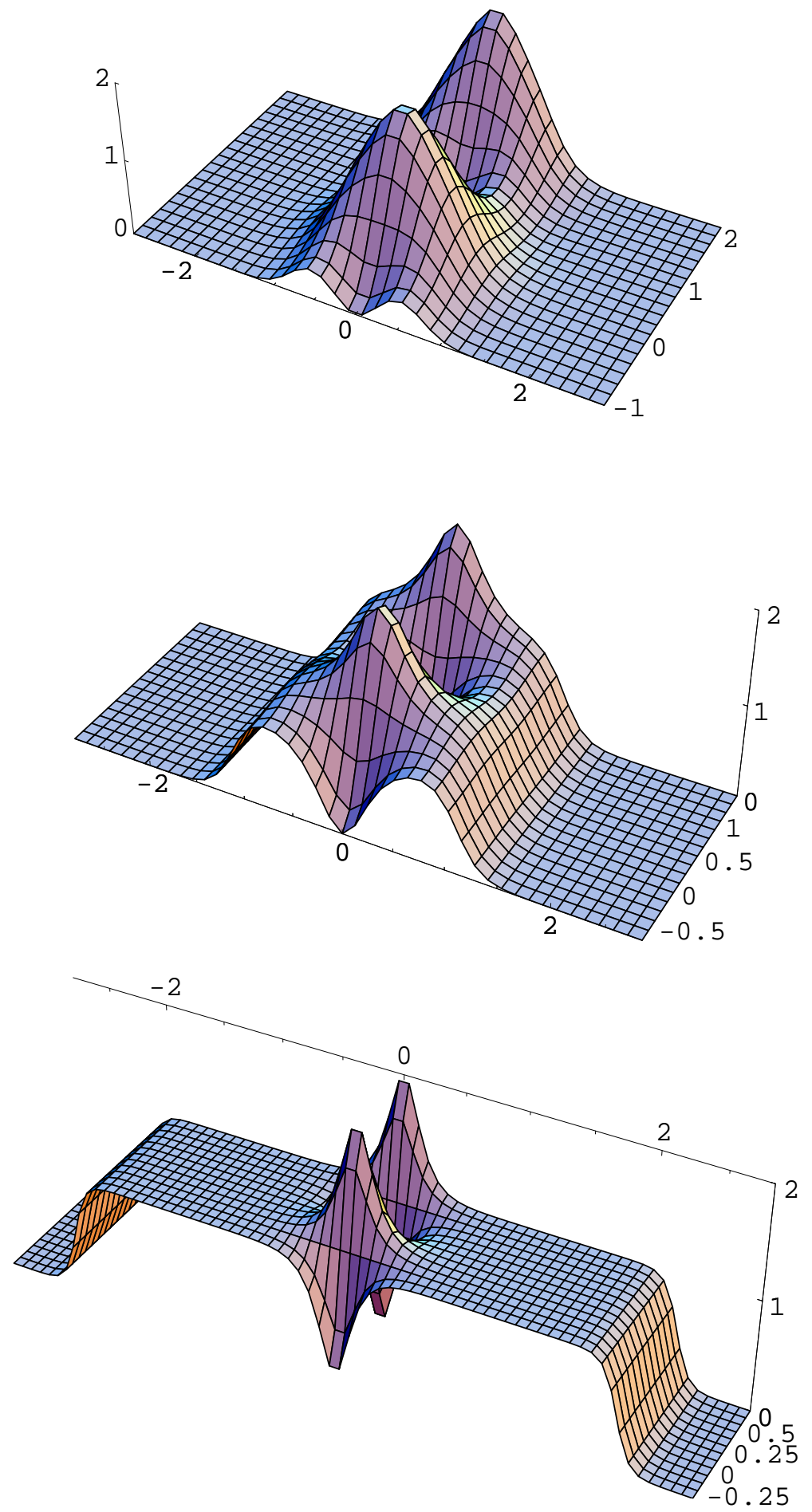

Fig. 5: The cylinder soliton (5.8) at various circumferences: $l=3$ (top), $l=2$ (middle), and $l=1$ (bottom). One and a half copies of the soliton are displayed. The axes are as in figure 2 . 


\section{3. $T^{2}$}

Just as in the case of the cylinder, a soliton on the torus can be represented as a lattice of solitons on the plane. With $\tau$ the modular parameter of the torus and $\sqrt{\theta} l$ its circumference in the $x^{1}$ direction, place the solitons in the $z$-plane at $z_{j_{1} j_{2}}=\left(j_{1}+j_{2} \tau\right) l / \sqrt{2}$, $j_{1}, j_{2} \in \mathbb{Z}$. Again, the forces between the soliton and its images will balance out, and the moduli space (which is the torus itself) is exact.

In principle there is no problem defining the projection operator whose image is the infinite dimensional hyperplane spanned by $\left\{\left|z_{j_{1} j_{2}}\right\rangle: j_{1}, j_{2} \in \mathbb{Z}\right\}$, for any size and shape of torus. In practice, however, we have only been able to find an explicit expression for the field configuration corresponding to such a projection operator in the special case where the parameter $A \equiv \tau_{2} l^{2} / 2 \pi$ (which is area of the torus in units of $2 \pi \theta$ ) is an integer. A major simplification occurs in this case because then the operators $U_{1} \equiv e^{-i l \hat{y}^{2}}$ and $U_{2} \equiv e^{i l\left(\tau_{2} \hat{y}^{1}-\tau_{1} \hat{y}^{2}\right)}$ generating the two lattice translations commute, and the same method as that used for the cylinder above can be employed. The details of the derivation are presented in appendix B; the final result is:

$$
\begin{aligned}
\phi(w, \bar{w})=\frac{\lambda}{2}( & \frac{\sum \vartheta_{00}^{*}\left(\nu-\frac{n}{A}\right) \vartheta_{00}\left(\nu+\frac{n}{A}\right)}{\sum\left|\vartheta_{00}\left(\nu+\frac{n}{A}\right)\right|^{2}}+\frac{\sum \vartheta_{00}^{*}\left(\nu-\frac{n+1 / 2}{A}\right) \vartheta_{00}\left(\nu+\frac{n+1 / 2}{A}\right)}{\sum\left|\vartheta_{00}\left(\nu+\frac{n+1 / 2}{A}\right)\right|^{2}} \\
& \left.+\frac{\sum \vartheta_{10}^{*}\left(\nu-\frac{n}{A}\right) \vartheta_{10}\left(\nu+\frac{n}{A}\right)}{\sum\left|\vartheta_{10}\left(\nu+\frac{n}{A}\right)\right|^{2}}+\frac{\sum \vartheta_{10}^{*}\left(\nu-\frac{n+1 / 2}{A}\right) \vartheta_{10}\left(\nu+\frac{n+1 / 2}{A}\right)}{\sum\left|\vartheta_{10}\left(\nu+\frac{n+1 / 2}{A}\right)\right|^{2}}\right),
\end{aligned}
$$

where $\nu \equiv \sqrt{2} w /(\sqrt{\theta} l)$, all theta functions take $\tau / A$ as their second argument, and all sums run from $n=0$ to $A-1$. It is not obvious how to generalize such a formula to the case where $A$ is not an integer. The shape of this soliton for the case $A=2$ is plotted in figure 6 .

Consider now the alternative case where $1 / A$ is an integer. Here the Moyal star product is in fact equivalent to ordinary pointwise multiplication of functions, and so a small puzzle arises: No non-trivial continuous solutions to the equation of motion $\phi \star \phi=\lambda \phi$ are possible, yet one can certainly define the projection operator $P$ whose image is the hyperplane spanned by $\left\{\left|z_{j_{1} j_{2}}\right\rangle: j_{1}, j_{2} \in \mathbb{Z}\right\}$. It is not hard to guess the resolution: this lattice of coherent states actually spans the entire Hilbert space, so $P$ is just the identity, and the "soliton" is the constant solution $\phi=\lambda$. Indeed, the formula (5.9) shows explicitly that this is the case when $A=1$. But we can say even more: it is known [32-34] that 

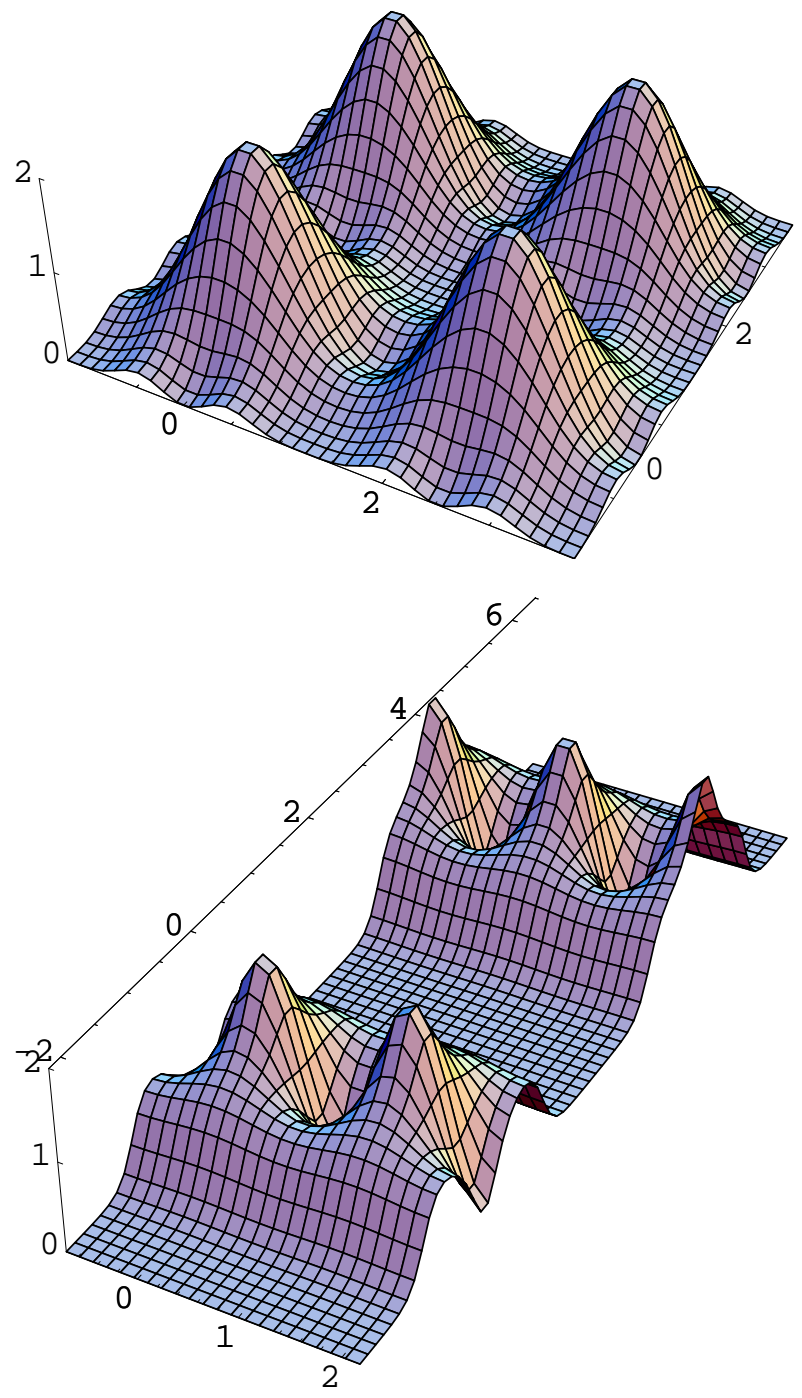

Fig. 6: The soliton (5.9) on the torus of area $4 \pi \theta(A=2)$ for two values of the modular parameter: the square torus $\tau=i$ (top), and $\tau=3 i+1 / 2$ (bottom). Four copies of the torus are displayed. The axes are as in figure 2.

any lattice of coherent states with $A \leq 1$ is a complete (actually, overcomplete) basis for the Hilbert space. 8 We thus conclude that there are no stable scalar solitons on any torus - rational or irrational — of area less than or equal to $2 \pi \theta$.

8 The case $A=1$ is known as a von Neumann lattice, since it was first discussed by him in [42], where the claim was made (without proof) that such a lattice forms a complete basis. An amusing fact about von Neumann lattices is that they are overcomplete by exactly one state, that is, there is exactly one linear relation among the infinitely many states. Lattices with $A<1$ are overcomplete by infinitely many states. 


\section{Solitons in higher dimensions}

The analysis of this paper easily generalizes to $2 d+1$ dimensional scalar field theory with spacelike noncommutativity, although we will see that the topological structure of the moduli space $\mathcal{M}_{k}^{d}$ in higher dimensions is much richer than in $d=1$.

We choose complex coordinates $w_{r}, r=1, \ldots, d$, in which the noncommutativity parameter takes the form $\theta^{r \bar{s}}=-i \delta_{r s} \theta_{s}$. The Weyl-Moyal correspondence maps the field $\phi$ to an operator $\hat{\phi}$ on the Hilbert space $\mathcal{H}^{d} \equiv \mathcal{H} \otimes \cdots \otimes \mathcal{H}$ of a particle in $d$ dimensions, with $w_{r} \rightarrow \sqrt{\theta_{r}} a_{r}$ and $\partial_{r} \rightarrow-\frac{1}{\sqrt{\theta_{r}}}\left[a_{r}^{\dagger}, \cdot\right]$. The moduli space at infinite $9 \theta$ again consists of operators of the form $\hat{\phi}=\lambda P$ for any projection operator $P$ of a given rank $k$. The Bogomolnyi bound (3.4) on the leading contribution from the kinetic energy takes the form

$$
\begin{aligned}
E_{1}\left[\hat{\phi}_{0}\right] & =(2 \pi)^{d} \lambda^{2} \theta \sum_{r=1}^{d} \frac{1}{\theta_{r}} \operatorname{Tr}\left[a_{r}, P\right]\left[P, a_{r}^{\dagger}\right] \\
& =(2 \pi)^{d} \lambda^{2} \theta \sum_{r=1}^{d} \frac{1}{\theta_{r}} \operatorname{Tr}\left(P+2 F_{r}(P)^{\dagger} F_{r}(P)\right) \\
& \geq(2 \pi)^{d} \lambda^{2} \theta k \sum_{r=1}^{d} \frac{1}{\theta_{r}},
\end{aligned}
$$

where $\theta \equiv \theta_{1} \cdots \theta_{d}$ and $F_{r}(P) \equiv(1-P) a_{r} P$. The moduli space $\mathcal{M}_{k}^{d}$ at finite $\theta$ is therefore the space of projection operators on $\mathcal{H}^{d}$ whose image is an invariant subspace of all of the $a_{r}$.

A large class of such operators may be constructed by letting $P$ project onto the space spanned by $k$ independent coherent states $\left|\vec{z}_{i}\right\rangle \equiv e^{\vec{z}_{i} \cdot \vec{a}^{\dagger}}|\overrightarrow{0}\rangle$. Such a $P$ corresponds to a multi-centered gaussian with peaks at $\vec{z}_{i}$. Naively, the moduli space of such solitons seems to be $\operatorname{Sym}^{k}\left(\mathbb{C}^{d}\right)$, as we saw is indeed the case in $d=1$. However, in dimension $d>1$ coincident solitons have more moduli than are present in the symmetric product, and we will see that the full moduli space $\mathcal{M}_{k}^{d}$ is the so-called Hilbert scheme Hilb ${ }^{k}\left(\mathbb{C}^{d}\right)$ of $k$ points in $\mathbb{C}^{d}$. Before introducing the general machinery of Hilbert schemes, we turn in the next subsection to the relatively simple case of $d=2$ in order to gain some insight into the geometry of the moduli space when higher dimensional solitons come together.

9 By this we mean the limit in which all of the $\theta_{r}$ are taken to infinity with their ratios held fixed, while the energy is rescaled by a factor of $m^{2} \theta_{1} \cdots \theta_{d}$, generalizing (2.3). 


\section{1. $4+1$ dimensions}

Consider first the case $k=2$, with two separated solitons described by the projection operator onto the space spanned by $\left|\vec{z}_{1}\right\rangle$ and $\left|\vec{z}_{2}\right\rangle$. When the two solitons come together we have

$$
\lim _{\vec{z}_{i} \rightarrow \vec{z}} \operatorname{span}\left\{\left|\vec{z}_{1}\right\rangle,\left|\vec{z}_{2}\right\rangle\right\}=\operatorname{span}\left\{|\vec{z}\rangle, \vec{\gamma} \cdot \vec{a}^{\dagger}|\vec{z}\rangle\right\}, \quad \text { where } \vec{\gamma} \equiv \lim _{\vec{z}_{i} \rightarrow \vec{z}} \frac{\vec{z}_{1}-\vec{z}_{2}}{\left|\vec{z}_{1}-\vec{z}_{2}\right|}
$$

Thus the "origin" of the relative moduli space is not a single point, but rather a $\mathbb{P}^{1}$ parametrized by the complex direction $\vec{\gamma}$ along which the two solitons came together. This is in contrast to the $d=1$ case studied in section 3, where we found that two solitons brought together along any direction end up at the unique point $\operatorname{span}\left\{|z\rangle, a^{\dagger}|z\rangle\right\}$.

The physical significance of the sphere hiding at the coincidence locus is made clear by applying the Weyl-Moyal correspondence to the solution $\hat{\phi}_{\vec{\gamma}}=\lambda P(\vec{\gamma})$, where $P(\vec{\gamma})$ is the projection operator onto $\operatorname{span}\left\{|\overrightarrow{0}\rangle, \vec{\gamma} \cdot \vec{a}^{\dagger}|\overrightarrow{0}\rangle\right\}$. This yields the function

$$
\phi_{\vec{\gamma}}(\vec{w})=16 \lambda e^{-2\left|w_{1}\right|^{2} / \theta_{1}-2\left|w_{2}\right|^{2} / \theta_{2}} \frac{\left|w_{1} \gamma_{1} / \sqrt{\theta_{1}}+w_{2} \gamma_{2} / \sqrt{\theta_{2}}\right|^{2}}{|\vec{\gamma}|^{2}},
$$

from which we see that the modulus $\vec{\gamma}$ encodes information about the shape of the level two lump. In other words, whereas two coincident solitons in $d=1$ have (other than the overall translational mode) only a modulus corresponding to separating the two solitons, in $d=2$ there is in addition a modulus corresponding to deforming the lump.

Factoring out the $\mathbb{C}^{2}$ center of mass, we can parametrize the relative moduli space with coordinates $z \in \mathbb{C}$ and $\vec{\gamma} \in \mathbb{C}^{2} \backslash\{\overrightarrow{0}\}$ by letting

$$
\operatorname{im} P(z, \vec{\gamma})=\operatorname{span}\{|z \vec{\gamma}\rangle,|-z \vec{\gamma}\rangle\}
$$

These coordinates are subject to the identification $(z, \vec{\gamma}) \sim(z / \lambda, \lambda \vec{\gamma})$ for $\lambda \in \mathbb{C} \backslash\{0\}$, which we recognize as defining the complex line bundle $\mathcal{O}(-1)$ over $\mathbb{P}^{1}$. The base $\mathbb{P}^{1}$ sits at $z=0$, which can be seen from $\lim _{z \rightarrow 0} \operatorname{im} P(z, \vec{\gamma})=P(\vec{\gamma})$ as defined above.

Plugging (6.4) into (2.9) gives the Kähler potential on the moduli space

$$
K^{\prime}=\ln \left(\frac{2 \sinh \left(2|z|^{2}|\vec{\gamma}|^{2}\right)}{|z|^{2}}\right)
$$

where the factor in the denominator arises as in (3.13) from the Jacobian of the transformation to a basis which remains nondegenerate as $z \rightarrow 0$. Note that the Kähler potential (6.5) induces the familiar Fubini-Study metric on the $\mathbb{P}^{1}$ at $z=0$. 
We conclude that two solitons in $4+1$ dimensions resolve the singular configuration space $\operatorname{Sym}^{2}\left(\mathbb{C}^{2}\right)$ into a smooth complex manifold with topology $\mathbb{C}^{2} \times \mathcal{O}_{\mathbb{P}^{1}}(-1)$ and a smooth Kähler metric given by (6.5). In particular, the $\mathbb{P}^{1}$ at the origin has finite area $(2 \pi)^{3} \lambda^{2} \theta_{1} \theta_{2}$.

For $k>2$ solitons in $4+1$ dimensions the topology of the the moduli space is more complicated. For example, for $k=3$ the relative moduli space has, in addition to the $\left(\mathbb{C}^{2} \backslash\{\overrightarrow{0}\}\right) \times \mathcal{O}_{\mathbb{P}^{1}}(-1)$ when any two solitons come together, a $\mathbb{P}^{1}$ nontrivally fibered over $\mathbb{P}^{1}$ at the "origin" of the relative moduli space where all three solitons merge. These moduli again correspond to deformations of the shape of the soliton, in contrast to the situation in $d=1$ where the only moduli (other than overall translations) of a collection of coincident solitons correspond to separating some of the solitons from each other.

As we will see in the next subsection, $\mathcal{M}_{k}^{2}$ has the same topology as the moduli space of $k \mathrm{U}(1)$ instantons on noncommutative $\mathbb{R}^{4}$ [37], which is a smooth complex manifold for any $k$. However, two important differences with that case warrant mention. Firstly, the metric on the scalar soliton moduli space $\mathcal{M}_{k}^{2}$ is only Kähler and not hyperkähler. Secondly, nothing in our analysis requires that the noncommutativity parameter $\theta$ in 4 dimensions be self-dual. Indeed, the precise values of $\theta_{1}$ and $\theta_{2}$ scale out of the problem - their only role is to set the scale of the physical coordinates $w_{r}$ with respect to the dimensionless moduli space coordinates $z_{i}$. The moduli space thus possesses an accidental $\mathrm{SU}(2)$ symmetry which is not possessed by the full theory when $\theta_{1} \neq \theta_{2}$.

\subsection{Hilbert schemes of points}

In this subsection we unify the discussion of the moduli space of $k$ solitons in $2 d+1$ dimensions by showing that $\mathcal{M}_{k}^{d}$ is isomorphic to a mathematical object known as the Hilbert scheme $\operatorname{Hilb}^{k}\left(\mathbb{C}^{d}\right)$ of $k$ points in $\mathbb{C}^{d}$.

$\operatorname{Hilb}^{k}\left(\mathbb{C}^{d}\right)$ is defined as the set of ideals $\mathcal{I}$ of codimension $k$ in the polynomial ring $\mathbb{C}\left[x_{1}, \ldots, x_{d}\right]$. The correspondence between projection operators and ideals is intuitively clear: if $f$ is a polynomial in some ideal $\mathcal{I}$ and $g$ is any polynomial, then the polynomial $f g$ is still in $\mathcal{I}$. Therefore the polynomials in an ideal $\mathcal{I}$ may be thought of roughly as projection operators from all of $\mathbb{C}\left[x_{1}, \ldots, x_{d}\right]$ into $\mathcal{I}$. The precise correspondence we demonstrate is motivated by a nearly identical correspondence between projection operators on a Fock space and ideals in polynomial rings (see for example [37,36]) which appears in the construction of noncommutative instantons on $\mathbb{R}^{4}$.

For any polynomial $f \in \mathbb{C}\left[x_{1}, \ldots, x_{d}\right]$, we may construct the ket

$$
|f\rangle \equiv f\left(a_{1}^{\dagger}, \ldots, a_{d}^{\dagger}\right)|\overrightarrow{0}\rangle \in \mathcal{H}^{d} .
$$


The one-to-one correspondence between $\operatorname{Hilb}^{k}\left(\mathbb{C}^{d}\right)$ and the moduli space $\mathcal{M}_{k}^{d}$ goes as follows. For an ideal $\mathcal{I} \subset \mathbb{C}\left[x_{1}, \ldots, x_{d}\right]$, we let $1-P$ be the projection operator onto (the closure of) the linear subspace spanned by $\{|f\rangle: f \in \mathcal{I}\}$. Conversely, given $P \in \mathcal{M}_{k}^{d}$, we may recover $\mathcal{I}=\left\{f \in \mathbb{C}\left[x_{1}, \ldots, x_{d}\right]: P|f\rangle=0\right\}$. This $\mathcal{I}$ is indeed an ideal by virtue of the fact that $P a_{r}^{\dagger}=P a_{r}^{\dagger} P$.

For example, if $P$ projects onto the subspace spanned by $k$ independent coherent states $\left|\vec{z}_{i}\right\rangle$, then the corresponding ideal is simply the set of all polynomials which vanish at the $k$ points $\vec{z}_{i}$. If $n$ of the points $\vec{z}_{i}$ come together at $\vec{z}$, then the ideal becomes the set of polynomials with an order $n$ zero at $\vec{z}$.

For $d=1$ we have simply $\operatorname{Hilb}^{k}(\mathbb{C}) \cong \mathbb{C}^{k}$, in agreement with the result of section 3 . For $k=2$ but arbitrary $d$, a trivial generalization of the argument in the previous subsection shows that the origin of the relative moduli space is not just a point but a whole $\mathbb{P}^{d-1}$. The total space $\mathcal{M}_{2}^{d}$ in this case is the center of mass $\mathbb{C}^{d}$ times the complex line bundle $\mathcal{O}(-1)$ over $\mathbb{P}^{d-1}$. For $d=2$ but arbitrary $k$, the Hilbert scheme $\operatorname{Hilb}^{k}\left(\mathbb{C}^{2}\right)$ is a smooth manifold of complicated topology which arises as the moduli space of $k \mathrm{U}(1)$ instantons on noncommutative $\mathbb{R}^{4}$ [35-38].

For $k>3$ and $d>2$, however, the Hilbert scheme is not smooth, and in fact it is not even a manifold, having in general several different branches of varying dimension. We present an example in the next subsection.

\subsection{An exotic example}

It is not difficult to construct exotic branches of the moduli space $\mathcal{M}_{k}^{d}=\operatorname{Hilb}^{k}\left(\mathbb{C}^{d}\right)$ for sufficiently large $k$ and $d$. Let us consider $k=12$ solitons in $d=8.10$ If the moduli space $\mathcal{M}_{12}^{8}$ only contained information about the location of 12 solitons free to move about in $\mathbb{C}^{8}$, then we would expect it to be 96-dimensional. However, we will now construct a 99-dimensional submanifold of $\mathcal{M}_{12}^{8}$ which opens up when all of the solitons coincide.

We work in a harmonic oscillator basis $\mathcal{H}^{8}=\operatorname{span}\left\{\left|n_{1}, \ldots, n_{8}\right\rangle: n_{r} \geq 0\right\}$. Let us denote by $|\alpha\rangle, \alpha=1, \ldots, 36$, the 36 basis vectors which have $\sum n_{r}=2$. Then for any three linearly independent 36-component vectors $w_{1}^{\alpha}, w_{2}^{\alpha}$ and $w_{3}^{\alpha}$, we can define the projection operator $P$ by

$$
\operatorname{im} P\left(w_{1}, w_{2}, w_{3}\right)=\operatorname{span}\left\{|\overrightarrow{0}\rangle,|1, \ldots, 0\rangle, \cdots,|0, \ldots, 1\rangle, w_{1}^{\alpha}|\alpha\rangle, w_{2}^{\alpha}|\alpha\rangle, w_{3}^{\alpha}|\alpha\rangle\right\},
$$

10 A nearly identical construction works in many other cases - in $d=3$, for example, with as few as $k=97$ solitons. 
with summation over the $\alpha$ indices implied. This operator projects onto a 12-dimensional subspace of $\mathcal{H}^{8}$ which is invariant under all of the $a_{r}$. Therefore $P \in \mathcal{M}_{12}^{8}$ for any three vectors $w$. These vectors represent the choice of a three dimensional subspace of the 36-dimensional space spanned by $|\alpha\rangle$, and hence they parametrize the Grassmannian

$\operatorname{Gr}\left(3, \mathbb{C}^{36}\right)$. Now $\operatorname{Gr}\left(3, \mathbb{C}^{36}\right)$ is 99 -dimensional, so (6.7) gives a 99 parameter family of projection operators inside of $\mathcal{M}_{12}^{8}$.

The branch parametrized by projection operators of the form (6.7) cannot be smoothly connected to the 96-dimensional branch where all of the solitons are separated. The analysis of the global structure of the $\mathrm{Hilb}^{k}\left(\mathbb{C}^{d}\right)$ for general $k>3$ and $d>2$, including details about how the various branches connect to each other, is a problem that mathematicians have only begun to tackle.

\section{Acknowledgements}

It is a pleasure to thank R. Glauber, T. Graber, D. Gross, K. Hori, S. Katz, D. Khosla, A. Mikhailov, S. Minwalla, A. Naqvi, N. Nekrasov, K. Oh, B. Pioline, S. Sethi, A. Strominger, L. Thorlacius, C. Vafa, A. Volovich, M. Wijnholt, and S. Zhukov for helpful conversations. We are especially grateful to S. Minwalla for comments on the manuscript. This work was supported in part by DOE grant DE-FG02-91ER40654 and the David and Lucile Packard Foundation. One of us (R.G.) would like to thank the I.T.P. for hospitality and acknowledge the support of NSF grant PHY99-07949.

\section{Appendix A. Completeness and smoothness of the finite $\theta$ moduli space}

In this appendix we prove the various statements made in section 3 about the topology and geometry of the moduli space $\mathcal{M}_{k}$ when $n$ points $z_{a}$ come together at a point $z$, with the other $k-n$ points fixed. Note that throughout this appendix we will use indices $a, b=1, \ldots, n$ while the indices $i, j$ will continue to run from 1 to $k$, as in the body of the paper.

The completeness of the moduli space follows trivially from the Hilbert scheme analysis of section 6 , but nevertheless it is useful to see explicitly how to construct a basis of states which is nondegenerate as two or more solitons come together. In particular, this is necessary to prove the assertion made above (3.13) that the Jacobian of the required change of basis is the Vandermonde determinant, and to verify that the metric obtained from (3.13) extends smoothly to the coincidence locus. 


\section{A.1. Some elementary machinery}

The proofs are completely straightforward but require some rather heavy notation, which we now introduce. Define $u_{a}=z_{a}-z$. We will make use of the $n \times n$ Vandermonde matrix $V$ with entries

$$
V_{a}^{b}=u_{a}^{b-1}
$$

which has determinant

$$
\Delta \equiv \operatorname{det} V=\prod_{a>b}\left(u_{a}-u_{b}\right)
$$

We will also make use of the polynomials defined by

$$
F_{\left\{c_{a}\right\}}\left(u_{a}\right)=\left|\begin{array}{ccc}
u_{1}^{c_{1}} & \cdots & u_{n}^{c_{1}} \\
\vdots & & \vdots \\
u_{1}^{c_{n}} & \cdots & u_{n}^{c_{n}}
\end{array}\right|
$$

where $\left\{c_{a}\right\}$ is a set of $n$ ordered nonnegative integers. It is clear that $F_{\left\{c_{i}\right\}}$ is a homogeneous polynomial in the $u_{i}$ which vanishes unless the $c_{i}$ are distinct. The lowest degree non-zero one is therefore just the Vandermonde determinant:

$$
F_{0, \ldots, n-1}\left(u_{a}\right)=\Delta
$$

Since the polynomial $F_{\left\{c_{a}\right\}}$ vanishes if any two of the $u_{a}$ are equal, it must be divisible by $u_{a}-u_{b}$ for all $a$ and $b$, and hence by $\Delta$. Therefore

$$
Q_{\left\{c_{a}\right\}}\left(u_{a}\right) \equiv F_{\left\{c_{a}\right\}}\left(u_{a}\right) / \Delta
$$

is again a homogeneous polynomial. Furthermore, since both $\Delta$ and $F_{\left\{c_{a}\right\}}$ are antisymmetric under the interchange of any two of the $u_{a}, Q_{\left\{c_{a}\right\}}$ must be a symmetric polynomial in the $u_{a}$. Now introduce an auxiliary variable $u$ and consider the determinant

$$
\Delta \prod_{a=1}^{n}\left(u+u_{a}\right)=\left|\begin{array}{cccc}
1 & 1 & \cdots & 1 \\
-u & u_{1} & \cdots & u_{n} \\
\vdots & \vdots & & \vdots \\
(-u)^{n} & u_{1}^{n} & \cdots & u_{n}^{n}
\end{array}\right|=\sum_{b=0}^{n} u^{b} F_{0, \ldots, \widehat{b}, \ldots, n}\left(u_{a}\right)
$$

where the notation $\widehat{b}$ means that the index $b$ is to be omitted. Upon dividing both sides of (A.6) by $\Delta$ we learn that the $Q_{\left\{c_{a}\right\}}$ of degree $\leq n$,

$$
\sigma_{b}\left(u_{a}\right) \equiv Q_{0, \ldots, \widehat{n-b}, \ldots, n}\left(u_{a}\right), \quad 0 \leq b \leq n
$$


are precisely the elementary symmetric polynomials in the $u_{a}$ :

$$
\begin{aligned}
\sigma_{0} & =1, \\
\sigma_{1} & =\sum_{a} u_{a}, \\
\sigma_{2} & =\sum_{a<b} u_{a} u_{b}, \\
& \vdots \\
\sigma_{n} & =\prod_{a=1}^{n} u_{a} .
\end{aligned}
$$

The $\sigma_{a}$ are good coordinates on $\operatorname{Sym}^{k}(\mathbb{C})$ in a neighborhood of $u_{a}=0$ and are therefore the coordinates of interest as we take $n$ points $z_{a}$ to $z$ keeping the other $k-n$ points fixed. Note that each $Q_{\left\{c_{a}\right\}}$ of degree greater than $n$ can be expressed as a polynomial in the $\sigma_{a}$.

The inverse of the Vandermonde matrix is

$$
\left(V^{-1}\right)_{b}^{a}=\frac{(-1)^{a+b}}{\Delta} F_{0, \ldots, \widehat{b}, \ldots, n}\left(u_{1}, \ldots, \widehat{u_{a}}, \ldots, u_{n}\right)
$$

Finally, we will use the identity

$$
\begin{aligned}
Q_{0, \ldots, \hat{b}, \ldots, n-1, p}\left(u_{a}\right) & =\frac{(-1)^{n}}{\Delta} \sum_{a=1}^{n}(-1)^{a} u_{a}^{p} F_{0, \ldots, \widehat{b}, \ldots, n-1}\left(u_{1}, \ldots, \widehat{u_{a}}, \ldots, u_{n}\right) \\
& =(-1)^{n+b} \sum_{a=1}^{n} u_{a}^{p}\left(V^{-1}\right)^{a}{ }_{b}
\end{aligned}
$$

which is obtained by expanding out the determinant defined by the left-hand side in the last row (the row containing $u_{a}^{p}$ ).

\section{A.2. Completeness}

We start with the basis $\left\{\left|z_{i}\right\rangle\right\}$. Expanding out the exponential in $\left|z_{a}\right\rangle=e^{u_{a} a^{\dagger}}|z\rangle$ gives

$$
\left|z_{a}\right\rangle=\sum_{b=1}^{n}|z ; b\rangle V^{b}{ }_{a}+\sum_{p=n+1}^{\infty}|z ; p\rangle u_{a}^{p-1}
$$

where

$$
|z ; m\rangle \equiv \frac{\left(a^{\dagger}\right)^{m-1}}{(m-1) !}|z\rangle
$$


Multiplying (A.11) by $V^{-1}$ gives

$$
\begin{aligned}
\left|z_{a}\right\rangle\left(V^{-1}\right)_{b}^{a} & =|z ; b\rangle+\sum_{p=n+1}^{\infty}|z ; p\rangle \sum_{a=1}^{n} u_{a}^{p-1}\left(V^{-1}\right)^{a}{ }_{b} \\
& =|z ; b\rangle+(-1)^{n+b} \sum_{p=n+1}^{\infty}|z ; p\rangle Q_{0, \ldots, \widehat{b}, \ldots, n-1, p-1}\left(u_{a}\right)
\end{aligned}
$$

using (A.10).

The polynomial multiplying $|p\rangle$ in (A.13) has degree greater than zero for any $p>n$, so that when we take all the $u_{a}$ to zero these terms vanish and we have simply

$$
\lim _{z_{a} \rightarrow z}\left|z_{a}\right\rangle\left(V^{-1}\right)^{a}{ }_{b}=|z ; b\rangle
$$

This establishes both (3.8) and the fact that the Jacobian of the necessary change of basis is the Vandermonde determinant, as advertised above (3.13).

\section{A.3. Smoothness}

Let $y_{i}=z_{i}-c$ be the relative coordinates on $\mathcal{M}_{k}$ as in section 3.3. Repeated application of the exponential expansion yields the formula

$$
\operatorname{det}\left(e^{\bar{y}_{i} y_{j}}\right)=\frac{1}{k !} \sum_{n_{1}, \ldots, n_{k}=0}^{\infty} \frac{1}{n_{1} ! \cdots n_{k} !}\left|F_{n_{1}, \ldots, n_{k}}\left(y_{1}, \ldots, y_{k}\right)\right|^{2}
$$

Since each $F$ is divisible by the Vandermonde determinant $\Delta=\prod_{i>j}\left(y_{i}-y_{j}\right)$ as in (A.5), the Kähler potential (3.13) is simply given by

$$
\begin{aligned}
e^{K^{\prime}} & =\frac{1}{k !} \sum_{n_{1}, \ldots, n_{k}=0}^{\infty} \frac{1}{n_{1} ! \cdots n_{k} !}\left|Q_{n_{1}, \ldots, n_{k}}\left(y_{1}, \ldots, y_{k}\right)\right|^{2} \\
& =\frac{1}{k !}\left[c_{k}(0)+\sum_{i=1}^{k} c_{k}(i)\left|\sigma_{i}(y)\right|^{2}+\mathcal{O}\left(\sigma_{i}^{3}\right)\right]
\end{aligned}
$$

where the $c_{k}(i)$ are some positive numbers. It is manifest from the second line of (A.16) that $K^{\prime}$ is well behaved as the solitons are brought together, i.e. as $\sigma_{i}(y) \rightarrow 0$. We see directly from this derivation how dividing $e^{K}$ by the Vandermonde determinant renders the Kähler potential $K^{\prime}$ nonsingular on the coincident locus. 


\section{Appendix B. Construction of the soliton on the integral torus}

We consider a torus with periodicities $l$ and $\tau l$ (in units of $\sqrt{\theta}$ ), where $A \equiv \tau_{2} l^{2} / 2 \pi$ is assumed to be an integer. The generators of the fundamental group are represented by the unitary operators

$$
U_{1} \equiv e^{-i l \hat{y}^{2}}, \quad U_{2} \equiv e^{i l\left(\tau_{2} \hat{y}^{1}-\tau_{1} \hat{y}^{2}\right)},
$$

which commute for such an integral torus. We wish to construct the projection operator whose image is spanned by the lattice of coherent states,

$$
U_{1}^{j_{1}} U_{2}^{j_{2}}|0\rangle, \quad\left(j_{1}, j_{2}\right) \in \mathbb{Z}^{2}
$$

Following the same strategy as on the cylinder, we will find a particular linear combination,

$$
|\psi\rangle=\sum_{j_{1}, j_{2}} c_{j_{1} j_{2}} U_{1}^{j_{1}} U_{2}^{j_{2}}|0\rangle
$$

that satisfies

$$
\left\langle\psi\left|U_{1}^{j_{1}} U_{2}^{j_{2}}\right| \psi\right\rangle=\delta_{j_{1} 0} \delta_{j_{2} 0} .
$$

The projection operator we seek is then

$$
P=\sum_{j_{1}, j_{2}} U_{1}^{j_{1}} U_{2}^{j_{2}}|\psi\rangle\langle\psi| U_{2}^{-j_{2}} U_{1}^{-j_{1}}
$$

We employ a generalization of the so-called $k q$ representation [34,43], which provides a basis of simultaneous eigenstates of $U_{1}$ and $U_{2}$ :

$$
|k q\rangle \equiv \sqrt{\frac{l}{2 \pi}} e^{-i \tau_{1}\left(\hat{y}^{2}\right)^{2} / 2 \tau_{2}} \sum_{j} e^{i j l k}|q+j l\rangle,
$$

where the ket on the right is a $\hat{y}^{1}$ eigenstate. We thus have

$$
U_{1}|k q\rangle=e^{-i l k}|k q\rangle, \quad U_{2}|k q\rangle=e^{i l \tau_{2} q}|k q\rangle .
$$

The set $\{|k q\rangle: 0 \leq k<2 \pi / l, 0 \leq q<l\}$ forms an orthonormal and complete basis for the Hilbert space. In terms of wave functions in the $k q$ representation, (B.3) becomes

$$
C_{\psi}(k, q) \equiv\langle k q \mid \psi\rangle=\sum_{j_{1}, j_{2}} c_{j_{1} j_{2}} e^{-i j_{1} l k+i j_{2} l \tau_{2} q}\langle k q \mid 0\rangle=\tilde{c}(k, q) C_{0}(k, q),
$$


where

$$
C_{0}(k, q) \equiv\langle k q \mid 0\rangle=\frac{1}{\pi^{1 / 4} \sqrt{l}} \exp \left(-\frac{\tau}{2 i \tau_{2}} k^{2}+i k q\right) \vartheta_{00}\left(\frac{q+k \tau / \tau_{2}}{l}, \frac{\tau}{A}\right) .
$$

Note that $\tilde{c}$ is doubly periodic: $\tilde{c}(k+2 \pi / l, q)=\tilde{c}(k, q+l / A)=\tilde{c}(k, q)$. The orthonormality condition (B.4) becomes

$$
\begin{aligned}
\delta_{j_{1} 0} \delta_{j_{2} 0} & =\int_{0}^{2 \pi / l} d k \int_{0}^{l} d q e^{-i j_{1} l k+i j_{2} l \tau_{2} q}\left|C_{\psi}(k, q)\right|^{2} \\
& =\int_{0}^{2 \pi / l} d k \int_{0}^{l / A} d q e^{-i j_{1} l k+i j_{2} l \tau_{2} q}|\tilde{c}(k, q)|^{2} \sum_{n=0}^{A-1}\left|C_{0}\left(k, q+n \frac{l}{A}\right)\right|^{2} .
\end{aligned}
$$

Hence (choosing $\tilde{c}$ real),

$$
C_{\psi}(k, q)=\frac{C_{0}(k, q)}{\sqrt{2 \pi / A \sum_{n=0}^{A-1}\left|C_{0}(k, q+n l / A)\right|^{2}}} .
$$

With $|\psi\rangle$ now in hand, we would like to find the field configuration corresponding to the projection operator (B.5). The inverse Weyl-Moyal transformation yields a Fourier expansion on the torus:

$$
\begin{aligned}
& \phi\left(y_{1}, y_{2}\right)= \\
& \quad \frac{\lambda}{A} \sum_{j_{1}, j_{2}}\left\langle\psi\left|\exp \left[\frac{2 \pi i}{l}\left(j_{1} \hat{y}^{1}+\frac{j_{2}-\tau_{1} j_{1}}{\tau_{2}} \hat{y}^{2}\right)\right]\right| \psi\right\rangle \exp \left[-\frac{2 \pi i}{l}\left(j_{1} y^{1}+\frac{j_{2}-\tau_{1} j_{1}}{\tau_{2}} y^{2}\right)\right] .
\end{aligned}
$$

To find the Fourier coefficients in terms of the $k q$ wave function of $|\psi\rangle$, we need the following result, which may be derived from (B.6):

$$
\begin{aligned}
\left\langle k^{\prime} q^{\prime}\right| \exp & {\left[\frac{2 \pi i}{l}\left(j_{1} \hat{y}^{1}+\frac{j_{2}-\tau_{1} j_{1}}{\tau_{2}} \hat{y}^{2}\right)\right]|k q\rangle=} \\
& \sum_{j} \exp \left(\frac{2 \pi i j_{1} q^{\prime}}{l}+\frac{\pi i j_{1} j_{2}}{A}+i j l k\right) \delta\left(q-q^{\prime}-\frac{j_{2} l}{A}+j l\right) \sum_{j^{\prime}} \delta\left(k-k^{\prime}-\frac{2 \pi j^{\prime}}{l}\right) .
\end{aligned}
$$

One can then show that

$$
\begin{gathered}
\phi\left(y^{1}, y^{2}\right)=\frac{\pi \lambda}{A} \sum_{j_{2}=0}^{2 A-1} e^{-2 \pi i j_{2} y^{2} /\left(\tau_{2} l\right)}\left(C_{\psi}^{*}\left(y^{2}, y^{1}-\frac{\tau_{1}}{\tau_{2}} y^{2}-\frac{j_{2} l}{2 A}\right) C_{\psi}\left(y^{2}, y^{1}-\frac{\tau_{1}}{\tau_{2}} y^{2}+\frac{j_{2} l}{2 A}\right)\right. \\
\left.+C_{\psi}^{*}\left(y^{2}+\frac{\pi}{l}, y^{1}-\frac{\tau_{1}}{\tau_{2}} y^{2}-\frac{j_{2} l}{2 A}\right) C_{\psi}\left(y^{2}+\frac{\pi}{l}, y^{1}-\frac{\tau_{1}}{\tau_{2}} y^{2}+\frac{j_{2} l}{2 A}\right)\right) .
\end{gathered}
$$

Combining this formula with (B.11) and $(\mathbb{B} .9)$, one finally arrives at the expression $(5.9)$. 


\section{References}

[1] E. Witten, "Noncommutative Geometry And String Field Theory," Nucl. Phys. B268 (1986) 253.

[2] N. Seiberg and E. Witten, "String Theory and Noncommutative Geometry," JHEP 09 (1999) 032, hep-th/9908142.

[3] E. Witten, "Noncommutative Tachyons and String Field Theory," hep-th/0006071.

[4] M. Schnabl, "String field theory at large B-field and noncommutative geometry," JHEP 11 (2000) 031, hep-th/0010034.

[5] R. Gopakumar, S. Minwalla and A. Strominger, "Noncommutative solitons," JHEP 05 (2000) 020, hep-th/0003160.

[6] K. Dasgupta, S. Mukhi and G. Rajesh, "Noncommutative tachyons," JHEP 06 (2000) 022, [hep-th/0005006].

[7] J. A. Harvey, P. Kraus, F. Larsen and E. J. Martinec, "D-branes and Strings as Noncommutative Solitons," JHEP 07 (2000) 042, [hep-th/0005031].

[8] A. Sen, "Descent Relations Among Bosonic D-branes," Int. J. Mod. Phys. A14 (1999) 4061, hep-th/9902105.

[9] A. Sen, "Universality of the Tachyon Potential," JHEP 12 (1999) 027, hepth/9911116].

[10] A. P. Polychronakos, "Flux tube solutions in noncommutative gauge theories," Phys. Lett. B495 (2000) 407, hep-th/0007043.

[11] D. Bak, "Exact Solutions of Multi-Vortices and False Vacuum Bubbles in Noncommutative Abelian-Higgs Theories," Phys. Lett. B495 (2000) 251, hep-th/0008204.

[12] M. Aganagic, R. Gopakumar, S. Minwalla and A. Strominger, "Unstable solitons in noncommutative gauge theory," JHEP 04 (2001) 001, hep-th/0009142.

[13] D. J. Gross and N. A. Nekrasov, "Solitons in noncommutative gauge theory," 03 (2001) 044, [hep-th/0010090].

[14] D. P. Jatkar, G. Mandal and S. R. Wadia, "Nielsen-Olesen Vortices in Noncommutative Abelian Higgs Model," JHEP 09 (2000) 018, hep-th/0007078.

[15] D. Bak and K. Lee, "Elongation of Moving Noncommutative Solitons," Phys. Lett. B495 (2000) 231, hep-th/0007107.

[16] A. S. Gorsky, Y. M. Makeenko and K. G. Selivanov, "On noncommutative vacua and noncommutative solitons," Phys. Lett. B492 (2000) 344, [hep-th/0007247].

[17] C. Zhou, "Noncommutative scalar solitons at finite $\theta$," hep-th/0007255].

[18] A. Solovyov, "On Noncommutative Solitons," Mod. Phys. Lett. A15 (2000) 2205, hep-th/0008199.

[19] J. A. Harvey, P. Kraus and F. Larsen, "Exact noncommutative solitons," JHEP 12 (2000) 024, hep-th/0010060]. 
[20] B. Durhuus, T. Jonsson and R. Nest, "Noncommutative scalar solitons: existence and nonexistence," Phys. Lett. B500 (2001) 320, [hep-th/0011139].

[21] M. Li, "Quantum Corrections to Noncommutative Solitons," hep-th/0011170.

[22] Y. Kiem, C. Kim and Y. Kim, "Noncommutative Q-balls," hep-th/0102160.

[23] M. G. Jackson, "The Stability of Noncommutative Scalar Solitons," [hep-th/0103217].

[24] U. Lindström, M. Roček and R. von Unge, "Non-commutative Soliton Scattering," JHEP 12 (2000) 004, hep-th/0008108.

[25] N. S. Manton, "A remark on the scattering of BPS monopoles," Phys. Lett. B110 (1982) 54.

[26] I. Bars, H. Kajiura, Y. Matsuo and T. Takayanagi, "Tachyon Condensation on Noncommutative Torus," Phys. Rev. D63 (2001) 086001, hep-th/0010101.

[27] E. M. Sahraoui and E. H. Saidi, "Solitons on compact and noncompact spaces in large noncommutativity," hep-th/0012259.

[28] E. J. Martinec and G. Moore, "Noncommutative Solitons on Orbifolds," hepth/0101199].

[29] D. Bak, K. Lee and J.-H. Park, "Noncommutative vortex solitons," [hep-th/0011099].

[30] K. Lee, D. Tong and S. Yi, "The moduli space of two U(1) instantons on noncommutative $R^{4}$ and $R^{3} \times S^{1}$," hep-th/0008092.

[31] K. Lee and P. Yi, "Quantum Spectrum of Instanton Solitons in five dimensional noncommutative $\mathrm{U}(N)$ theories," hep-th/9911186].

[32] A. M. Perelomov, "On the completeness of a system of coherent states," Teor. Mat. Fiz. 6 (1971) 213.

[33] V. Bargmann, P. Butera, L. Girardello and J. R. Klauder, "On the completeness of the coherent states," Rep. on Math. Phys. 2 (1971) 221.

[34] H. Bacry, A. Grossman and J. Zak, "Proof of completeness of lattice states in the $k q$ representation," Phys. Rev. B12 (1975) 1118.

[35] H. Nakajima, "Lectures on Hilbert schemes of points on surfaces," American Mathematical Society University Lecture Series, 1999.

[36] K. Furuuchi, "Instantons on Noncommutative $R^{4}$ and Projection Operators," Prog. Theor. Phys. 103 (2000) 1043, hep-th/9912047.

[37] N. A. Nekrasov, "Noncommutative instantons revisited," hep-th/0010017.

[38] H. W. Braden, N. A. Nekrasov, "Instantons, Hilbert Schemes and Integrability," hepth/0103204.

[39] Talk by R. Gopakumar at Strings 2001, Mumbai, http://theory.tifr.res.in/strings/Proceedings/gkumar/.

[40] O. Lechtenfeld, A. D. Popov, B. Spendig, "Noncommutative solitons in open N=2 string theory," hep-th/0103196.

[41] L. Hadasz, U. Lindström, M. Roček, R. von Unge, "Noncommutative Multisolitons: Moduli Spaces, Quantization, Finite $\theta$ Effects, and Stability," hep-th/0104017. 
[42] J. von Neumann, Mathematical Foundations of Quantum Mechanics, Princeton (1955).

[43] J. Zak, in Solid State Physics, edited by H. Ehrenreich, F. Seitz, and D. Turnbull (Academic, New York, 1972), Vol. 27. 